CWP-142P

DOE/ER/14079-30

November 1993

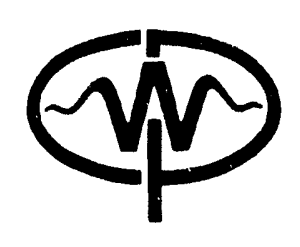

\title{
Elimination of numerical dispersion in finite-difference modeling and migration by flux-corrected transport
}

\author{
Tong Fei \\ Department of Mathematics \\ and \\ Ken Larner \\ Department of Geophysics
}

\section{DISCLAIMER}

\begin{abstract}
This report was prepared as an account of work sponsored by an agency of the United States Government. Neither the United States Government nor any agency thereof, nor any of their employees, makes any warranty, express or implied, or assumes any legal liability or responsibility for the accuracy, completeness, or usefulness of any information, apparatus, product, or process disclosed, or represents that its use would not infringe privately owned rights. Reference herein to any specific commercial product, process, or service by trade name, trademark, manufacturer, or otherwise does not nocessarily constitute or imply its endorsement, recommendation, or favoring by the United States Government or any agency thereof. The views and opinions of authors expressed berein do not necessarily state or reflect those of the United States Government or any agency thereof.
\end{abstract}

Center for Wave Phenomena

Colorado School of Mines

Golden, Colorado 80401

(303) 273-3557 


\title{
Elimination of numerical dispersion in finite-difference modeling and migration by flux-corrected transport
}

\author{
Tong Fei* and Ken Larner ${ }^{\dagger}$
}

\begin{abstract}
Finite-difference acoustic-wave modeling and reverse-time depth migration based on the full wave equation are general approaches that can take into account arbitary variations in velocity and density, and can handle turning waves well. However, conventional finite-difference methods for solving the acousticwave equation suffer from numerical dispersion when too few samples per wavelength are used. Here, we present two flux-corrected transport (FCT) algorithms, one based the second-order equation and the other based on first-order wave equations derived from the second-order one. Combining the FCT technique with conventional finite-difference modeling or reverse-time wave extrapolation can ensure finite-difference solutions without numerical dispersion even for shock waves and impulsive sources. Computed two-dimensional migration images show accurate positioning of reflectors with greater than 90 -degree dip. Moreover, application to real data shows no indication of numerical dispersion. The FCT correction, which can be applied to finite-difference approximations of any order in space and time, is an efficient alternative to use of approximations of increasing order.
\end{abstract}

\section{INTRODLCTION}

Finite-difference methods. following the approaches of Claerbout (1985), have been widely implemented for wave extrapolation in modeling and migration. Those approaches employ a one-way wave equation that allows energy to propagate either downward or upward, but not both. Although successful in many situations, such methods are limited by assumptions made in deriving the one-way wave equation. Moreover, finite-difference schemes based on the one-way wave equation contain a limit on the maximum dip angle of the reflector. To deal with a variable velocity

* Center for Wave Phenomena, Department of Mathematics and Computer Science, Colorado School of Mines, Golden, CO 80401

tCenter for Wave Phenomena. Department of Geophysics, Colorado School of Mines, Golden, CO 80401 
field, Kosloff and Baysal (1983) developed a two-dimensional migration scheme in the frequency and space domain based on a direct integration in depth of the acoustic wave equation.

Since finite-difference modeling and migration based on the full acoustic-wave equation have no additional physical assumptions, the finite-difference method potentially can address many issues, such as arbitrary velocity variation, turning waves, and multiple reflected waves. This method, however is costiy, placing heavy demands on computer memory and input/output devices (Reshef and Kessler, 1989; Blacquiere et al., 1989; Hale and Witte, 1992).

Conventional finite-difference schemes for numerically solving the wave equation suffer from undesirable ripples. so-called grid dispersion or numerical dispersion, particularly near large gradients in wavefields or when too coarse a computation grid is used. Alford et al. (1974) and Kelly et al. (1976) have studied the grid dispersion existing in finite-difference methods. They concludel that to eliminate the numerical dispersion, at least 11 and 5.5 points per half-power wavelength must be used for the second-order and fourth-order finite-difference methods, respectively. Consistent with their conclusions, Dablain (1986) stated that 8 and 4 grid points at the Nyquist frequency are required to eliminate dispersion for the second-order and fourth-order finite-difference methods, respectively.

The flux-corrected transport (FCT) method developed by Boris and Book (1973) and Book et al. (1975), has been successfully applied in the solution of the cortinuity equation in hydrodynamics in circumstances involving large gradients and discontinuities, where conventional algorithms fail. Even with use of coarse grids, the FCT algorithm can treat strong gradients, shocks and impulses without the usual dispersively generated ripples.

Here, we present and apply the FCT finite-difference technique to forward seismic modeling and reverse-time depth migration. The FCT approach is applicable in either of the two forms. The first FCT algorithm, which directly follow the hydrodyuamics approach, is based on the first-order partial differential equatin 3 rerived from the second-order wave equation through a change of dependent wris wes, whe the second FCT approach can be directly used with the second-order saver futiror. These FCT

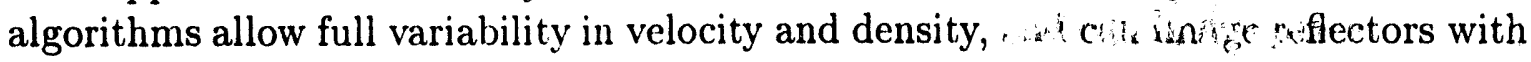
a wide range of dips, with no numerical dispersion. Whe read on atively coarse grid, with less computation they achieve accuracy comp rabis at conventional finite-difference approaches on a finer grid.

\section{THEORY}

For velocity and density ficlds that are functions of space, tile acoustic-wave equation is 


$$
\frac{1}{\rho} \frac{\partial^{2} P}{\partial^{2} t}=c^{2}\left[\frac{\partial}{\partial x}\left(\frac{1}{\rho} \frac{\partial P}{\partial x}\right)+\frac{\partial}{\partial y}\left(\frac{1}{\rho} \frac{\partial P}{\partial y}\right)+\frac{\partial}{\partial z}\left(\frac{1}{\rho} \frac{\partial P}{\partial z}\right)\right]+f(x, y, z, t),
$$

where $\rho=\rho(x, y, z)$ is density, $c=c(x, y, z)$ is wave velocity, and $f(x, y, z, t)$ is the source function.

This wave equation for inhomogencous media can be approximated by an explicit, conventional finite-difference scheme. However, this finite-difference discretization causes the phase speed to become a function of discretization interval, and generates undesirable dispersion when the spatial grid is too coarse (that is, too few samples per wavelength are used). The FCT technique is a correction procedure applied to the finite-difference result at each time step.

First, let us follow the original hydrodynamics approach by defining new dependent variables in the second-order acoustic wave equation so as to form four first-order, conservative. equations in these new variables.

With new dependent variables defined as

$$
\begin{gathered}
q \equiv \frac{1}{\rho c^{2}} \frac{\partial P}{\partial t} \\
u \equiv \frac{\partial P}{\partial x} \\
v \equiv \frac{\partial P}{\partial y} \\
w \equiv \frac{\partial P}{\partial z}
\end{gathered}
$$

the second-order acoustic wave equation (1) is reduced to the new first-order partial differential equation,

$$
\frac{\partial q}{\partial t}=\frac{\partial}{\partial x}\left(\frac{u}{\rho}\right)+\frac{\partial}{\partial y}\left(\frac{v}{\rho}\right)+\frac{\partial}{\partial y}\left(\frac{w}{\rho}\right)+f
$$

which is of the conservative form, and three additional first-order partial differential equations are derived from definitions (2) through (J),

$$
\begin{aligned}
& \frac{\partial u}{\partial t}=\frac{\partial}{\partial x}\left(\rho c^{2} q\right), \\
& \frac{\partial v}{\partial t}=\frac{\partial}{\partial y}\left(\rho c^{2} q\right),
\end{aligned}
$$




$$
\frac{\partial w}{\partial t}=\frac{\partial}{\partial z}\left(\rho c^{2} q\right)
$$

As in the hydrodynamics problem. the FCT correction can then be applied to these first-order equations. Aftcr we applied the FCT to the first-order system, we found out that the FCT correction could readily be applied to the second-order wave equation, as well.

Conceptually, the FCT technique consists of two major stages - a conventional finite-difference stage (Stage I), followed by a correction stage (Stage II) which consists of diffusion and anti-diffusion corrections. Applied to a conventional finite-difference scheme of any order approximation in differencing, FCT ideally would entail application of a corrective diffusion localized to just those regions where non-physical ripples tend to form. This diffusion step would be carried out in a conservative way; that is, whenever a portion of the wavefield is removed at one point, the same amount is inserted somewhere else, and, as a result, there is no net loss or gain of the quantity (e.g., pressure) to the system. In real situations, if there is no a priori information about where the dispersion might exist, the FCT attempts to apply the diffusion everywhere (steps 2 and 4 in Appendix A and B). Therefore, once the solution is diffused, an opposing anti-diffusion is introduced to reduce the diffusion wherever it seems not to be needed (steps 3,5 and 6 in Appendix A and B).

The Appendix A gives the procedure for solving equations (6) through (9) by the flux-corrected transport finite-difference method, while Appendix B gives the FCT correction procedure for solving second-order wave cquation (1).

In the computer implementation here, we apply the absorbing boundary condition of Clayton and Engquist (1980) to the side boundaries and the lower boundary.

\section{NUMERICAL EXAMPLES}

We have tested the FCT correction on one-dimensional forward problems, twodimensional modeling and reverse-time depth migration. The reverse-time depth migration, which basically is the same as forward nodeling, simply runs time backwards.

For the one-dimensional case, where $\frac{\partial}{\partial x}=\frac{\partial}{\partial y}=0$, forward modeling tests involve a wavelet that is an isolated full-cycle of a sinusoid, $0.5 \cos (2 \pi f t)+0.5$, as well as for a rectangle function with different widths. The medium is homogeneous in these tests, and the FCT algorithm used is based on the first-order partial differential equations.

For the two-dimensional case where $\frac{\partial}{\partial y}=0$, we do tests of both modeling and migration with FCT correction based on both the first-order partial differential equations and the second-order wave equation. 


\section{One-dimensional tests}

In the one-dimensional tests. the medium has a constant density and a constant velocity of $2 \mathrm{~km} / \mathrm{s}$. In cach of the modeling tests, we specify a time sequence at the surface and examine a snapshot of the wavefield generated in depth. Figures 1 through 3 show the snapshot:s at $1 \mathrm{~s}$, generated by three isolated sinusoids at the surface 0.333 -s apart. The frequency for these tests is $10 \mathrm{~Hz}$. The spatial steps are 0.01 $\mathrm{km}$ (twenty samples per wavelength), $0.025 \mathrm{~km}$ (eight samples per wavelength) and $0.04 \mathrm{~km}$ (five samples per wavelength), respectively. Similarly, Figures 4 through 6 show the snapshots at $1 \mathrm{~s}$ for rectangle-function pulses at the surface with spatial step $0.01 \mathrm{~km}$, and with eight, four and two samples per pulse width, respectively.

In each of the six figures, we compare the results for the ideal solution with those obtained by a standard finitc-difference method and by the FCT algorithm. The ideal solution has a waveform that is unchanged during propagation. In Figure 1, the fine-grid simulation (large dominant wavelength relative to $\Delta x$ ), the standard leapfrog method applied to first-order equations gives a good result with only a small amount of numerical dispersion. while the dispersion-caused ripples are absent in the FCT solution.

For the coarse grids, eight samples per wavelength (Figure 2) and five samples per wavelength (Figure 3), the standard leapfrog mothod yields a progressively more distorted and weakened waveform, with large precursor oscillations, and significant loss in resolution. The FCT correction still produces a good waveform shape, without the ripples, but still with amplitude losses of about 8 percent in Figure 2 and about 15 percent (for the deepest cvent) in Figure 3. The weakened amplitudes are the result of applying diffusion and anti-diffusion in the FCT method; the anti-diffusion did not fully correct for the amplitude loss in the diffusion stage. The results suggest that the FCT correction can give the correct position of the wavefront, as well as greatly reduced numerical dispersion. While it partly improves the resolution, even when few samples per wavelength are used, the loss in amplitude in finite-difference result is not fully recovered by the FCT correction.

For the rectangle pulses (Figures 4 through 6) with spatial step of $0.01 \mathrm{~km}$, the standard leapfrog finite-difference method gives poor solutions for all choices of sampling, whereas the FCT solutions show no numerical dispersion. Also for the choices of eight and four samples per pulse width, the FCT method gives the correct amplitudes (Figures 4 and 5) because relatively lower frequencies are dominant in these two cases than in tests with the sinusoidal pulses. The waveform with the FCT correction has only little distortion since, for the one-dimensional case, it is easy to control the diffusion and anti-diffusion coefficients ( $\eta_{1}$ and $\eta_{2}$, see appendices) to obtain a good waveform. For the narrowest rectangle pulse, with only two samples per pulse width (Figure 6), the FCT correction greatly improves the pulse shape and restores resolution, but it falls short in restoring the significant loss of amplitude.

From these one-dimensional tests, we see that the FCT technique can treat large gradients and shocks without the usual ripple artifacts. The FCT algorithm not only 
removes the grid-dispersion errors, it also yields a waveform with little distortion. Moreover, the FCT can recover some, but not all, amplitude loss when too few samples per wavelength (or pulse width) are used.

\section{Reflector model}

The model shown in Figure $T$ has five reflectors, each with a horizontal segment and a segment with dip varying from 30 degrees to 90 degrees, in 15-degree increments. The input zero-offset time section for each of two different velocity models (Figure 8) was obtained by a Kirchhoff modeling program. The first velocity model has velocity linearly increasing with depth. and the second has a velocity gradient in a sloping direction. Figures 9 and 10 show the results of FCT reverse-time depth migration applied to each of the zcro-offset synthetic data sets in Figure 8. The spatial steps, $\Delta x$ and $\Delta z$, in these tests are both $0.008 \mathrm{~km}$, and the time step $\Delta t$ is chosen to satisfy a conventional stability condition. The input zero-offset synthetic data contain a symmetric Ricker wavelet with dominant frequency of $20 \mathrm{~Hz}$.

Here, a leapfrog finite-difference method, second-order accuracy both in time and space, is first used to obtain the solution for the first-order partial differential equations, and then the FCT correction is applied. For these tests, the number of grid points $G_{0}$ per upper half-power wavelength is about 6 (at the position with the smallest velocity), which gives a relatively coarse grid according to Kelly's criterion for the second-order accuracy method. Numerical dispersion is present in the solutions without FCT correction (not shown here). However, with the FCT correction, the migration images are dispersion free.

Figure 8a shows the synthetic data for velocity linearly increasing with depth, $c(z)=1.6+0.6 z \mathrm{~km} / \mathrm{s}$. The migrated section (Figure 9) indicates that the FCT method can accurately image reflectors with all dips. The broadening of deeper events is caused by the increasing of velocity with depth.

For a velocity field that is changing both vertically and horizontally, $c(x, z)=1.5+$ $0.2 x+0.35 z \mathrm{~km} / \mathrm{s}$, the migrated section (Figure 10) again shows accurate positioning of the horizontal and dipping reflectors. For the vertical reflector, the amplitude appears weak because some of the unmigrated data fall out of the recording window (Figure 8b).

\section{Wedge model}

Figure 11 shows a two-dimensional model in which the velocity in a rectangular area is assumed to be zero, while outside that area the velocity is a constant 2.438 $\mathrm{km} / \mathrm{s}$. The modeled area in the tests is $5.266 \times 5.266 \mathrm{~km}^{2}$, with upper left corner at the origin. The upper left corner of the rectangular is at $(2.194 \mathrm{~km}, 3.510 \mathrm{~km})$ and a line source is located at $(2.633 \mathrm{~km}, 3.072 \mathrm{~km})$. The time variation of the source function is $(t-0.1) e^{-\alpha(t-0.1)^{2}}$, where $t$ is time, measured in $\mathrm{s}$, and $\alpha=700 \mathrm{~s}^{-2}$. This gives a upper half-power frequency of $10 \mathrm{~Hz}$, and the corresponding half-power wavelength is about $0.244 \mathrm{~km}$ for velocity $v=2.438 \mathrm{~km} / \mathrm{s}$. 
For the fine grid, $\triangle x=0.02194 \mathrm{~km}$, the number of grid points $G_{0}$ per upper halfpower wavelength is about 11. Figure 12a shows the snapshot at $1.026 \mathrm{~s}$ for the wavefield computed by the second-order conventional finite-difference method, and Figure 12b shows the snapshot at the same time for the FCT-corrected wavefield. For this fine sampling, both the conventional finite-difference result and the FCTcorrected result are accurate, and show no numerical dispersion.

On a medium-coarseness grid, $\triangle x=0.04388 \mathrm{~km}$. for which $G_{0} \approx 5.5$, the wavefield obtained by the second-order conventional finite-difference approach becomes dispersive (Figure 12c). The FCT correction, however, removes the numerical dispersion (Figure 12d). Note also in the conventional solution (Figure 12c) some loss of resolution, which is partially recovered with the FCT correction.

When the grid size becomes more coarse, $\Delta x=0.08776 \mathrm{~km}$, for which $G_{0} \approx 2.7$, the oscillations in the conventional finite-difference solution increase significantly (Figure 12e). The FCT correction, however. still removes the numerical dispersion (Figure 12f). In addition to the numerical dispersion. use of too coarse a grid in the conventional modeling has caused considerable wavelet distortion, loss of amplitude, and, in places, apparent time errors (note the slightly non-circular wavefront at the depth between $4 \mathrm{~km}$ and $5 \mathrm{~km}$ ). Although the grid dispersion is removed by the FCT correction, the use of too coarse a grid causes loss of amplitude and resolution (as would happen in an attenuating medium) that cannot be fully corrected by the FCT method.

\section{Salt-dome structure model}

Figure 13 shows a reflector structure modeling the boundary of an overhanging salt dome with dip as large as 125 degrees. The input zero-offset time section for velocity linearly increasing with depth $(c(z)=1.5+0.9 z \mathrm{~km} / \mathrm{s})$ was obtained by a Kirchhoff modeling program. The wavelet is a symmetric Ricker wavelet with dominant frequency of $30 \mathrm{~Hz}$. Figures 14 through 18 show the reverse-time depth-migration results by the second-order and the fourth-order conventional finite-difference methods, without and with the FCT correction.

For Figure 14, a migration image by second-order conventional finite-differencing, the number of grid points per upper half-power wavelength at depth $z=0 \mathrm{~km}$, where velocity is $1.5 \mathrm{~km} / \mathrm{s}$, is about 3.7 (coarse grid). The migration image shows strong numerical dispersion. To overcome this problem. we can use a finer grid, at high computational cost and heavy use of computer memory for the second-order method, or we can use a higher-order scheme. Figure 15 shows the migration image by fourthorder conventional finite-differencing on the same grid. As expected, the fourth-order method significantly reduces the numerical dispersion and also improves the resolution. Since this spatial grid is still coarse for the fourth-order method, a significant amount of dispersion remains. With use of a finer grid $\left(G_{0} \approx 5.5\right.$ at $\left.z=0 \mathrm{~km}\right)$, the migration image for the fourth-order method becomes dispersion-free (not shown here).

The FCT technique can be equally applied to the higher-order finite-difference 
results. Figures 16 through 18 show the FCT-corrected results by the second-order and fourth-order methods.

In Figure 16 (same grid size as for Figure 14), the FCT correction has removed the numerical dispersion seen in Figure 14, and has slightly improved the resolution. But, since the grid is too coarse for the second-order method, the FCT correction has not completely cured the resolution problem. For the finer grid with $G_{0} \approx 5.5$ at $z=0$ $\mathrm{km}$ (still a coarse grid for the conventional second-order method), the FCT correction on the second-order finite-difference yields good resolution, again along with removal of the numerical dispersion (Figure 17). Applying the FCT correction to the fourthorder method on the coarser grid (Figure 18), also eliminates the numerical dispersion and yields the desired resolution (compare with Figures 14 through 16).

\section{Field data}

Figure 19 shows a DMO-stacked section from the Gulf of Mexico. The migrated section by the fourth-order conventional finite-difference method, with spatial step size of $6 \mathrm{~m}$, is shown in Figure 20. The part of the image inside the dashed box is enlarged in Figure 21. For comparison, Figure 22 shows a closeup view of a comparable portion of the unmigrated D.MO-stacked section. The arrows in Figures 21 and 22 point to comparable positions on the two sections, where reflections have little slope and therefore should not be changed much by the migration. The added reflection events at the arrow in Figure 21 might therefore be artifacts of numerical dispersion. With the FCT correction (Figure 23), the suspect events are removed.

\section{EFFICIENCY}

Just as increasing the order of the finite-difference approximation increases the amount of computation on a fixed grid, so does use of the added steps in FCT correction. As with use of higher-order finite-differences, however, the FCT correction allows computation on a coarser grid.

For 2-D problems, the second-order conventional finite-difference method for solving equation (1) requires about $k N^{3}$ computations (Table 1), assuming that the number of grid points in the horizontal and vertical directions, and the number of time steps are each equal to $N$. Here, $k$ is a fixed proportionality constant. The secondorder finite-difference method for solving equations (6), (7) and (9) increases the amount of computation to about $1.6 \mathrm{kN}^{3}$, which is nearly the same as that for the fourth-order finite-difference method for solving equation (1). The diffusion and antidiffusion steps in the FCT method for the first-order equations again increase the computational effort, to about $4 k N^{3}$; similarly, the FCT correction for the secondorder wave equation increases the computation to about $2.8 \mathrm{kN}$ for the second-order finite-difference method. and to about $3.4 k N^{3}$ for the fourth-order finite-difference method. But the FCT method gains in efficiency because fewer points per wavelength are required for acceptable accuracy in terms of both numerical dispersion and 
resolution. In practice, at least 11 points per upper half-power wavelength must be used for the second-order conventional finite-difference method and 5.5 points must be used for the fourth-order method to achieve acceptable accuracy. In contrast, for the FCT method, only 5.5 and 3.7 points need to be used for the second-order and fourth-order methods, respectively, to obtain comparable accuracy. Thus, as seen in Table 1, the FCT method should gain in speed compared with the conventional second-order and fourth-order finite-difference methods. For 3-D problems, the increases in efficiency and speed of the FCT method should be even greater.

\begin{tabular}{|c|c|c|c|}
\hline Method & $\begin{array}{c}\text { Computational cost } \\
\text { proportional to }\end{array}$ & $\begin{array}{c}\text { Points per } \\
\text { wavelength }\left(G_{0}\right)\end{array}$ & $\begin{array}{c}\text { Relative } \\
\text { speed }\end{array}$ \\
\hline $\begin{array}{c}\text { 2nd-order eqn; } \\
\text { 2nd-order FD }\end{array}$ & $k N^{3}$ & 11 & 1 \\
\hline $\begin{array}{c}\text { 1st-order eqns; } \\
\text { 2nd-order FD }\end{array}$ & $1.6 k N^{3}$ & 11 & 0.63 \\
\hline $\begin{array}{c}\text { 2nd-order eqn; } \\
\text { 4th-order FD }\end{array}$ & $1.6 k N^{3}$ & 5.5 & 5 \\
\hline $\begin{array}{c}\text { 2nd-order eqn; } \\
\text { 2nd-order FD with FCT }\end{array}$ & $2.8 k N^{3}$ & 5.5 & 2.9 \\
\hline $\begin{array}{c}\text { 1st-order eqns; } \\
\text { 2nd-order FD with FCT }\end{array}$ & $4 k N^{3}$ & 5.5 & 2.0 \\
\hline $\begin{array}{c}\text { 2nd-order eqn; } \\
\text { 4th-order FD with FCT }\end{array}$ & $3.4 k N^{3}$ & 3.7 & 7.7 \\
\hline
\end{tabular}

Table 1. Relative computation speeds with and without FCT.

As seen in Table 1, solution of the second-order wave equation is more efficient than that of the first-order equations. However, it is easier to code the FCT corrections for the first-order system in applications to elastic media (Fei, 1993). Note also in Table 1, the speed of the fourth-order conventional finite-difference method exceeds that of the second-order finite-difference method with the FCT correction. If one were to go to the next higher-order (sixth-order) finite-difference method, the higher-order finite-difference would also be somewhat faster than the fourth-order finite-difference with the FCT correction. However, the higher-order finite-difference effort can still fail to completely remove the numerical dispersion where velocity is particularly low, locally.

The speeds of the FCT correction listed on the table pertain to corrections that are applied everywhere throughout the computation area of interest. However, where velocity has large contrast, the numerical dispersion tends to be restricted to regions with small velocity, and we can choose to localize the FCT correction to those regions. With such localization of the FCT effort, the FCT approach can be particularly efficient. For a constant velocity medium, no such benefit of localization of action is available. 


\section{CONCLUSIONS}

As we have seen. the FCT method provides an efficient and effective means of overcoming problems of numerical dispersion that arise in standard finite-difference algorithms if too few samples per wavelength are used. Even for wavefields with strong gradients and discontinuities, the FCT method can still produce solutions without numerical dispersion. Also, the migration results presented here show that the FCT method can produce good images of reflectors with dip beyond 90 degrees for velocity that increases with depth.

The flux-corrected transport correction, initially applied to the first-order equations of hydrodynamics. may be applied to the acoustic wave equation written either as a second-order equation or as a set of first-order equations. While the FCT applied to the first-order system is casier to code for elastic-wave extrapolation in anisotropic media (Fei, 1993), for the acoustic problem the second-order equation is more efficiently treated by finite-difference methods. The use of the FCT correction imposes no limitation on the spatial variation of the velocity and density fields for either modeling or reverse-time depth migration. The additional FCT steps add about 1.5 times more computation for the first-order equations, and about 1.8 times more for the second-order wave equation, than that of the stanclard second-order explicit finitedifferencing step. The FCT method, however. offers the opportunity to use a coarse grid (fewer samples per wavelength) to obtain accuracy in wavefield extrapolation that is comparable to that obtained by conventional finite-difference methods. The use of next higher-order finite-difference methods also allows the use of a coarse grid, and with slightly less computation effort than that for the lower-order finite-difference with the FCT correction. However, in situations where higher-order finite-difference methods cannot effectively remove all the numerical dispersion, the FCT correction is an alternative technique that can ensure a dispersion-free solution.

When using higher-order methods in the finite-differencing step, the relative computation cost of the FCT is reduced, since the FCT correction is independent of the order of finite-differencing. Despite these benefits of the FCT corrections, the computation effort of reverse-time depth migration is still about three to ten times (depending on the velocity model) that of the Kirchhoff integral method for 2-D migration. For 3-D migration and modeling, the computational effort of any finitedifference method (the FCT method in particular) is proportional to $N^{4}$, while that of the Kirchhoff integral method or Gaussian beam method is proportional to $N^{5}$, suggesting that the FCT method might be more competitive with the Kirchhoff integral method or Gaussian beam method for 3-D problems.

\section{ACKNOWLEDGMENTS}

We thank Dave Hale for helpful discussions and comments, and gratefully acknowledge the support by the members of the Consortium Project on Seismic Inverse Methods for Complex Structures at the Center for Wave Phenomena, Colorado School 
of Mines. This work was also partially supported by the United States Department of Energy, grant number DE-FG02-89ER14079.

\section{REFERENCES}

Alford, R., Kelly, K., and Boore. D., 1974, Accuracy of finite-difference modeling of the acoustic wave equation: Geophysics, 39, 834-842.

Blacquiere, G., Debeye, H.. Wapenaar, C., and Berkhout, A., 1989, 3D table-driven migration: Geophys. Prosp., 37, 925-958.

Boris, J. P., and Book, D. L., 1973, Flux-corrected transport. I. SHASTA, A fluid transport algorithm that works: J. Comput. Pliys., 11, 38-69.

Book, D. L., Boris, J. P. and Hain, K., 1975, Flux-corrected transport II: generalization of the method: J. Comput. Phys., 18, 248-283.

Claerbout, J., 1985. Imaging of the earth's interior: Blackwell Scientific Publications, Inc.

Clayton, R., and Engquist, B., 1980, Absorbing side boundary conditions for wave equation migration: Geophysics, 45, 895-904.

Dablain, M. A. 1986, The application of high-order differencing to the scalar wave equation: Geophysics, 51, 54-66.

Fei, T., 1993, Seismic modeling in anisotropic media: Center for Wave Phenomena Report, CWP-137, 177-196, Colorado School of Mines.

Hale, D., and Witte, D., 1992, Migration: progress and predictions: Presented at the 62nd Ann. Internat. Mtg. Soc. Expl. Geophys., Expanded Abstracts, 610-612.

Kelly, K., Ward, R., Treitel, S., and Alford, R., 1976, Synthetic seismograms: a finite-difference approach: Geophysics, 41, 2-27.

Kosloff, D. and Baysal, E., 1983, Migration with the full acoustic wave equation: Geophysics, 48, 677-687.

Reshef, M. and Kessler, D., 1989, Practical implementation of three-dimensional poststack depth migration: Geophysics, 54, 309-318.

\section{APPENDIX A: FLUX-CORRECTED TRANSPORT FOR THE FIRST-ORDER WAVE-EQUATION SYSTEM}

The flux-corrected transport (FCT) method (Boris and Book, 1973; Book, et al., 1975) was developed primarily for solving the first-crder continuity equation in hydrodynamics. To apply the FCT method to the first-order system for seismic wavefield extrapolation in acoustic media, we need to solve equations (6) through (9). By direct extension of the FCT approach in Boris and Book (1973), the FCT algorithm proceeds as follows: 
1. Advance the solutions on a staggered grid by a standard finite-difference method (leapfrog algorithm has been applied here) and obtain $q_{i, j, k}^{n+1}, u_{i+1 / 2, j, k}^{n+1}, v_{i, j+1 / 2, k}^{n+1}$ and $w_{i, j, k+1 / 2}^{n+1}$ at time level $n+1$. (For example, $q_{i, j, k}^{n}$ is the value of $q$ at the time sample $n, x$-coordinate sample $i, y$-coordinate sample $j$ and $z$-coordinate sample $k$ ). To save computation cost, the FCT correction (next few steps) need be performed only on the variable $q$. Such a correction is not necessary for computation of $u$. $v$ and $w$. Once $q$ is corrected, $u, v$ and $w$ can be computed directly from equations $(7)$ through $(9)$.

2. Compute diffusive fluxes at time level $n-1$ :

$$
\begin{aligned}
& Q_{x_{i+1 / 2, j, k}^{n-1}}^{n-1}=\eta_{1}\left(q_{i+1, j, k}^{n-1}-q_{i, j, k}^{n-1}\right) \\
& Q_{y_{i, j+1 / 2, k}^{n-1}}^{n-1}=\eta_{1}\left(q_{i, j+1, k}^{n-1}-q_{i, j, k}^{n-1}\right) \\
& Q_{z_{i, j, k+1 / 2}^{n-1}}^{n-1}=\eta_{1}\left(q_{i, j, k+1}^{n-1}-q_{i, j, k}^{n-1}\right),
\end{aligned}
$$

where $0 \leq \eta_{1} \leq 1$ is a coefficient that varies with position. In typical applications, $\eta_{1}$ ranges between 0.01 and 0.1 . The function can be determined from a few small-scale numerical experiments by considering the amplitude treatment for horizontal events. Results are not critically sensitive to the choice of $\eta_{1}$.

3. Compute diffusive fluxes at time level $n+1$, for use in step 6 below :

$$
\begin{aligned}
& \tilde{Q}_{x i+1 / 2, j, k}^{n+1}=\eta_{2}\left(q_{i+1, j, k}^{n+1}-q_{i, j, k}^{n+1}\right) \\
& \tilde{Q}_{y_{i, j+1 / 2, k}^{n+1}}^{n+1}=\eta_{2}\left(q_{i, j+1, k}^{n+1}-q_{i, j, k}^{n+1}\right) \\
& \tilde{Q}_{z i, j, k+1 / 2}^{n+1}=\eta_{2}\left(q_{i, j, k+1}^{n+1}-q_{i, j, k}^{n+1}\right),
\end{aligned}
$$

where $0 \leq \eta_{2} \leq 1$. The values of $\eta_{2}$ may differ from those of $\eta_{1}$. To preserve resolution, we generally use $\eta_{2}$ about 10 to 15 percent larger than $\eta_{1}$.

4. Modify (i.e. diffuse) the solution $q$ using $Q_{x}, Q_{y}$ and $Q_{z}$; this process smooths the solution (also causes an undesirable loss of amplitude) and eliminates the ripples caused by grid dispersion:

$$
\begin{aligned}
\tilde{q}_{i, j}^{n+1}=q_{i, j}^{n+1} & +\left(Q_{x_{i+1 / 2, j, k}}^{n-1}-Q_{x_{i-1 / 2, j, k}}^{n-1}\right) \\
& +\left(Q_{y_{i, j+1 / 2, k}^{n-1}}-Q_{y_{i, j-1 / 2, k}}^{n-1}\right) \\
& +\left(Q_{z_{i, j, k+1 / 2}^{n-1}}-Q_{z_{i, j, k-1 / 2}^{n-1}}^{n-1}\right)
\end{aligned}
$$

5. Take the differences of the diffused $\tilde{q}$ :

$$
\begin{aligned}
& X_{i+1 / 2, j, k}=\tilde{q}_{i+1, j, k}^{n+1}-\tilde{q}_{i, j, k}^{n+1} \\
& Y_{i, j+1 / 2, k}=\tilde{q}_{i, j+1, k}^{n+1}-\tilde{q}_{i, j, k}^{n+1} \\
& Z_{i, j, k+1 / 2}=\tilde{q}_{i, j, k+1}^{n+1}-\tilde{q}_{i, j, k}^{n+1} .
\end{aligned}
$$


6. Anti-diffuse the solution as follows, and obtain the corrected solution for $q$ :

$$
\begin{aligned}
q_{i, j, k}^{n+1}=i_{i, j, k}^{n+1} & -\left(X_{i+1 / 2, j, k}^{c}-X_{i-1 / 2, j, k}^{c}\right)-\left(Y_{i, j+1 / 2, k}^{c}-Y_{i, j-1 / 2, k}^{c}\right) \\
& -\left(Z_{i, j, k+1 / 2}^{c}-Z_{i, j, k-1 / 2}^{c}\right)
\end{aligned}
$$

where

$$
\begin{aligned}
& X_{i+1 / 2, j, k}^{c}=S_{x} \max \left\{0, \min \left[S_{x} X_{i-1 / 2, j, k}, \operatorname{abs}\left(\tilde{Q}_{x i+1 / 2, j, k}^{n+1}\right), S_{x} X_{i+3 / 2, j, k}\right]\right\}, \\
& Y_{i, j+1 / 2, k}^{c}=S_{y} \max \left\{0, \min \left[S_{y} Y_{i, j-1 / 2, k}, \operatorname{abs}\left(\tilde{Q}_{y_{i, j+1 / 2, k}}^{n+1}\right), S_{y} Y_{i, j+3 / 2, k}\right]\right\}, \\
& Z_{i, j, k+1 / 2}^{c}=S_{z} \max \left\{0, \min \left[S_{z} Z_{i, j, k-1 / 2}, \operatorname{abs}\left(\tilde{Q}_{z i, j, k+1 / 2}^{n+1}\right), S_{z} Z_{i, j, k+3 / 2}\right]\right\}, \\
& S_{x}=\operatorname{sign}\left\{\dot{Q}_{x_{i+1 / 2, j, k}}^{n+1}\right\}, \\
& S_{y}=\operatorname{sign}\left\{\dot{Q}_{y_{i, j+1 / 2, k}}^{n+1}\right\}, \\
& S_{z}=\operatorname{sign}\left\{\dot{Q}_{i, i, j, k+1 / 2}^{n+1}\right\} .
\end{aligned}
$$

\section{APPENDIX B: FLUX-CORRECTED TRANSPORT FOR THE SECOND-ORDER WAVE EQUATION}

To apply the FCT method to the second-order acoustic wave equation (1), the procedure is much the same as that discussed in Appendix A. The diffusive and antidiffusive fluxes, however, are computed differently. In equation (2), note that variable $q$ is the time derivative of variable $P$. Therefore, the fluxes for $P$ can be obtained by finite-differencing in time after finite-differencing in space. The algorithm proceeds as follows:

1. Advance the solutions by a standard second-order finite-difference method and obtain $P_{i, j, k}^{n+1}$ at time lcvel $n+1\left(P_{i, j, k}^{n+1}\right.$ is the value of $P$ at time sample $n+1$, $x$-coordinate ample $i, y$-coordinate sample $j$ and $z$-coordinate sample $k$ ).

2. Compute diffusive fluxes at time level $n$ :

$$
\begin{aligned}
& Q_{x i+1 / 2, j, k}^{n}=\eta_{1}\left(P_{i+1, j, k}^{n}-P_{i, j, k}^{n}-P_{i+1, j, k}^{n-1}+P_{i, j, k}^{n-1}\right) \\
& Q_{y_{i, j+1 / 2, k}^{n}}^{n}=\eta_{1}\left(P_{i, j+1, k}^{n}-P_{i, j, k}^{n}-P_{i, j+1, k}^{n-1}+P_{i, j, k}^{n-1}\right) \\
& Q_{z i, j, k+1 / 2}^{n}=\eta_{1}\left(P_{i, j, k+1}^{n}-P_{i, j, k}^{n}-P_{i, j, k+1}^{n-1}+P_{i, j, k}^{n-1}\right),
\end{aligned}
$$

where $0 \leq \eta_{1} \leq 1$ is a coefficient chosen as described for equation (A-1).

3. Compute diffusive fluxes at time level $n+1$, for use in step 6 below :

$$
\begin{aligned}
& \tilde{Q}_{x i+1 / 2, j, k}^{n+1}=\eta_{2}\left(P_{i+1, j, k}^{n+1}-P_{i, j, k}^{n+1}-P_{i+1, j, k}^{n}+P_{i, j, k}^{n}\right) \\
& \tilde{Q}_{y_{i, j+1 / 2, k}}^{n+1}=\eta_{2}\left(P_{i, j+1, k}^{n+1}-P_{i, j, k}^{n+1}-P_{i, j+1, k}^{n}+P_{i, j, k}^{n}\right) \\
& \tilde{Q}_{i i, j, k+1 / 2}^{n+1}=\eta_{2}\left(P_{i, j, k+1}^{n+1}-P_{i, j, k}^{n+1}-P_{i, j, k+1}^{n}+P_{i, j, k}^{n}\right),
\end{aligned}
$$


where $0 \leq \eta_{2} \leq 1$ is as described for equation (A-2).

4. Modify (i.e. diffuse) the solution $P$ using $Q_{x}, Q_{y}$ and $Q_{z}$; this process smooths the solution (also again causes some loss of amplitude) and eliminates the dispersive ripples:

$$
\begin{aligned}
\tilde{P}_{i, j}^{n+1}=P_{i, j}^{n+1} & +\left(Q_{x_{i+1 / 2, j, k}}^{n-1}-Q_{x_{i-1 / 2, j, k}}^{n-1}\right) \\
& +\left(Q_{y_{i, j+1 / 2, k}^{n-1}}-Q_{y_{i, j-1 / 2, k}}^{n-1}\right) \\
& +\left(Q_{z_{i, j, k+1 / 2}}^{n-1}-Q_{z_{i, j, k-1 / 2}^{n-1}}\right)
\end{aligned}
$$

5. Compute diffusive fluxes with the diffused $\tilde{P}^{n+1}$ and $P^{n}$ :

$$
\begin{aligned}
& X_{i+1 / 2, j, k}=\left(\tilde{P}_{i+1, j, k}^{n+1}-P_{i+1, j, k}^{n}\right)-\left(\tilde{P}_{i, j, k}^{n+1}-P_{i, j, k}^{n}\right) \\
& Y_{i, j+1 / 2, k}=\left(\tilde{P}_{i, j+1, k}^{n+1}-P_{i, j+1, k}^{n}\right)-\left(\tilde{P}_{i, j, k}^{n+1}-P_{i, j, k}^{n}\right) \\
& Z_{i, j, k+1 / 2}=\left(\tilde{P}_{i, j, k+1}^{n+1}-P_{i, j, k+1}^{n+1}\right)-\left(\tilde{P}_{i, j, k}^{n+1}-P_{i, j, k}^{n}\right) .
\end{aligned}
$$

6. Anti-diffuse the solution as follows, and obtain the corrected solution for $P$ :

$$
\begin{aligned}
P_{i, j, k}^{n+1}=\tilde{P}_{i, j, k}^{n+1} & -\left(X_{i+1 / 2, j, k}^{c}-X_{i-1 / 2, j, k}^{c}\right)-\left(Y_{i, j+1 / 2, k}^{c}-Y_{i, j-1 / 2, k}^{c}\right) \\
& -\left(Z_{i, j, k+1 / 2}^{c}-Z_{i, j, k-1 / 2}^{c}\right)
\end{aligned}
$$

where $X_{i+1 / 2, j, k}^{c}, Y_{i, j+1 / 2, k}^{c}$ and $Z_{i, j, k+1 / 2}^{c}$ are given in step 6 of Appendix A. 


\section{LIST OF FIGURES}

Fig. 1. One-dimensional test for isolated wavelets, each of which is a full-cycle of a sinusoid with a frequency of $10 \mathrm{~Hz}$, for a constant velocity of $2 \mathrm{~km} / \mathrm{s}$ and a constant density. The spatial step is $\Delta x=0.01 \mathrm{~km}$ (twenty samples per wavelength). (a) Idcal wavefield. (b) Wavefield obtained by a standard leapfrog finite-difference method. (c) Wavefield obtained with the FCT correction.

Fig. 2. Same as Figure 1, except $\triangle x=0.025 \mathrm{~km}$ (eight samples per wavelength).

Fig. 3. Same as Figure 1, except $\Delta x=0.04 \mathrm{~km}$ (five samples per wavelength).

Fig. 4. One-dimensional test for isolated rectangle pulses (eight samples per pulse width), for a constant velocity of $2 \mathrm{~km} / \mathrm{s}$ and a constant density. (a) Ideal wavefield. (b) Wavefield obtained by standard leapfrog finite-difference method. (c) Wavefield obtained with the FCT correction.

Fig. 5. Same as Figure 4, but here the isolated rectangle pulses have four samples per pulse width.

Fig. 6. Same as Figure 4, but here the isolated rectangle pulses have two samples per pulse width.

Fig. 7. Reflector model used to generate synthetic data for the tests described in Figures 9 and 10.

Fig. 8. Zero-offset synthetic time sections generatcd by Kirchhoff modeling for (a) velocity linearly increasing with depth, (b) velocity model with linear variation in $(x, z)$.

Fig. 9. Migrated section for velocity model $c(z)=1.6+0.6 z \mathrm{~km} / \mathrm{s}$, and constant density.

Fig. 10. Migrated section for velocity model $c(x, z)=1.5+0.2 x+0.35 z \mathrm{~km} / \mathrm{s}$, and constant density.

Fig. 11. Wedge model showing the source position and velocity field (rectangular void in a homogenous medium).

Fig. 12. Wavefield snapshot for different grid sizes. (a) and (b) are for fine grid $\left(G_{0} \approx\right.$ 11 ). (c) and (d) are for medium-coarseness grid $\left(G_{0} \approx 5.5\right)$. (e) and (f) are for coarse grid $\left(G_{0} \approx 2.7\right)$. (a), (c) and (e) are for the conventional finite-difference method. (b), (d) and (f) are the solution with the FCT correction.

Fig. 13. Reflector model used to generate synthetic data for the tests described in Figures 14 through 18. 
Fig. 14. Second-order conventional finite-difference migration for grid step sizes $\Delta x$ $=\Delta z=0.008 \mathrm{~km}\left(G_{0} \approx 3.7\right.$ at $\left.\mathrm{z}=0 \mathrm{~km}\right)$.

Fig. 15. Fourth-order conventional finite-difference migration for the same grid as that for Figure 14.

Fig. 16. Second-order finite-difference migration with the FCT correction for the same grid as that for Figure 14.

Fig. 17. Second-order finitc-difference migration with the FCT correction for grid step sizes $\Delta x=\Delta z=0.0055 \mathrm{~km}\left(G_{0} \approx 5.5\right.$ at $\left.\mathrm{z}=0 \mathrm{~km}\right)$.

Fig. 18. Fourth-order fiaite-difference migration with tne FCT correction for the same grid size as that for Figure 14.

Fig. 19. DMO and stacked section from Gulf of Mexico.

Fig. 20. Migrated section by the fourth-order conventional finite-difference method.

Fig. 21. Enlarged portion of migrated section by the fourth-order conventional finitedifference method.

Fig. 22. Closer view of DMO and stacked section from Gulf of Mexico.

Fig. 23. Enlarged portion of migrated section by fourth-order finite-difference method with the FCT correction. 


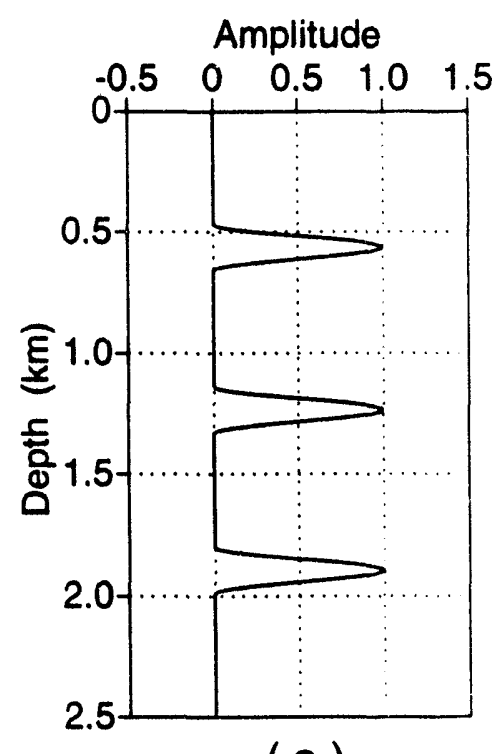

(a)
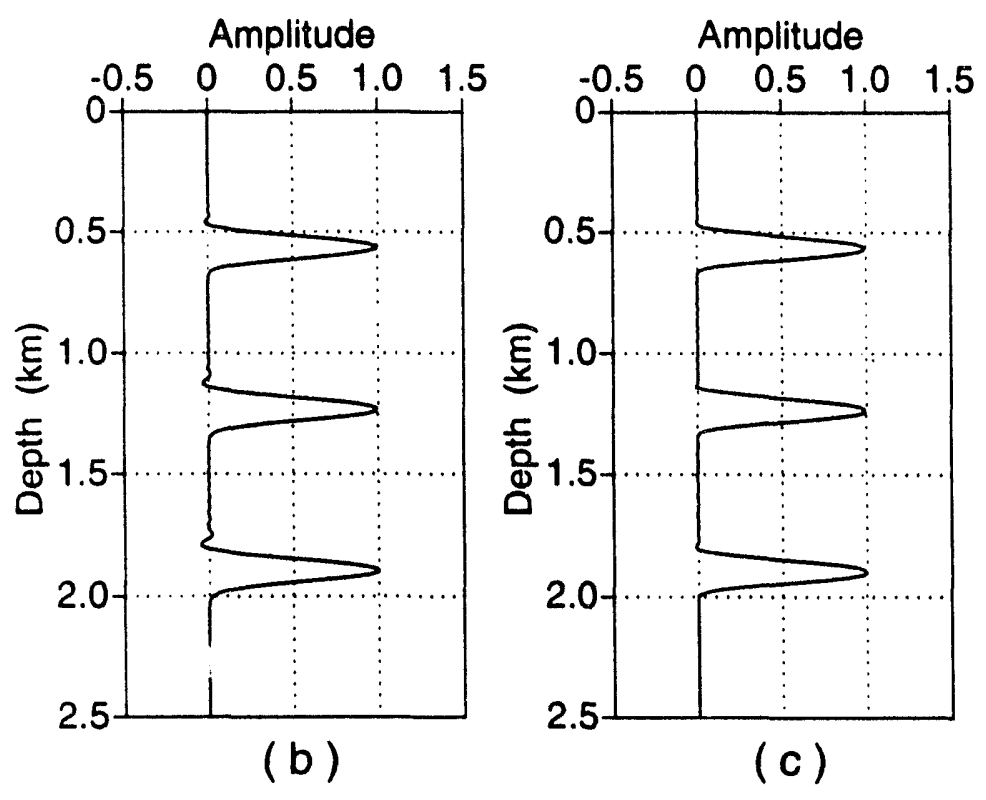

Fig. 1. 

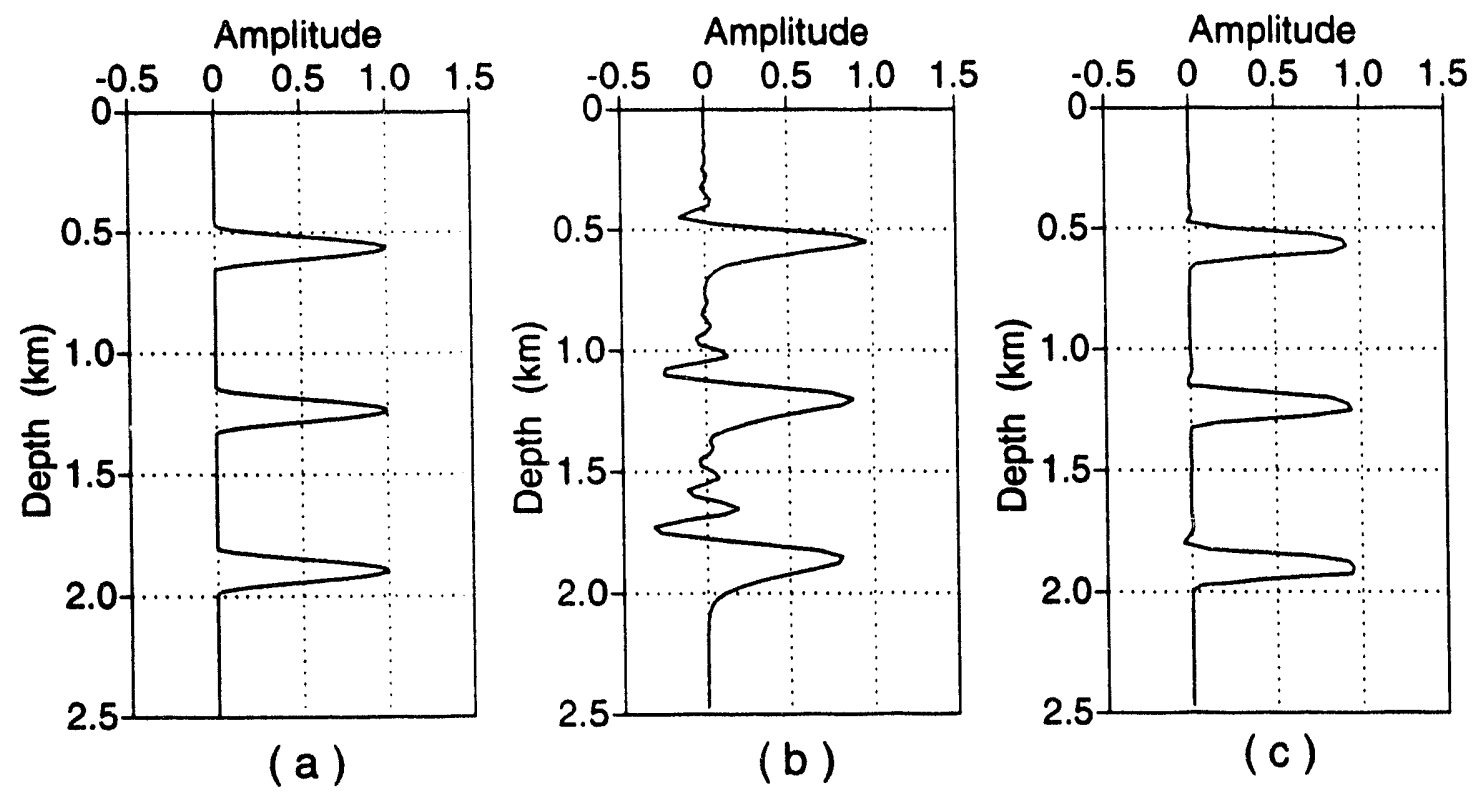

FIG. 2. 


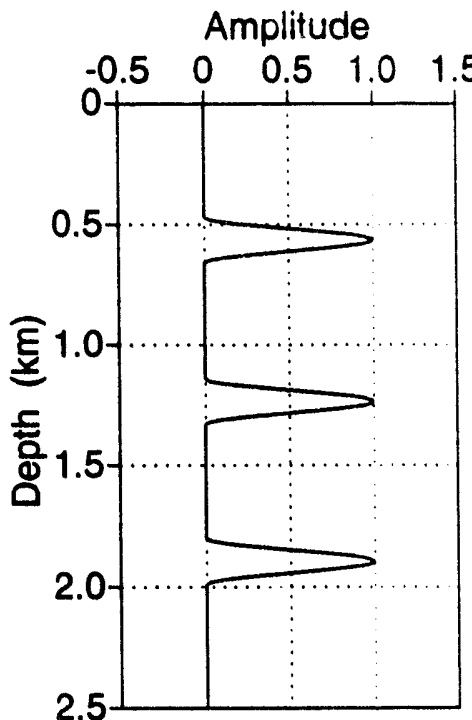

(a)

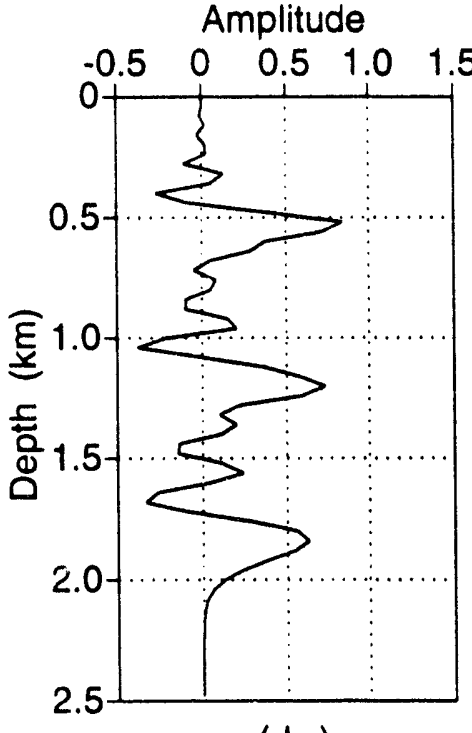

(b)

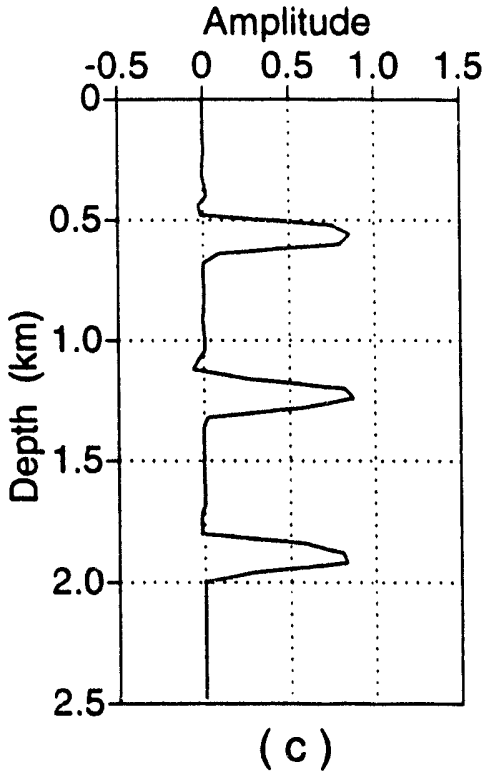

FIG. 3. 


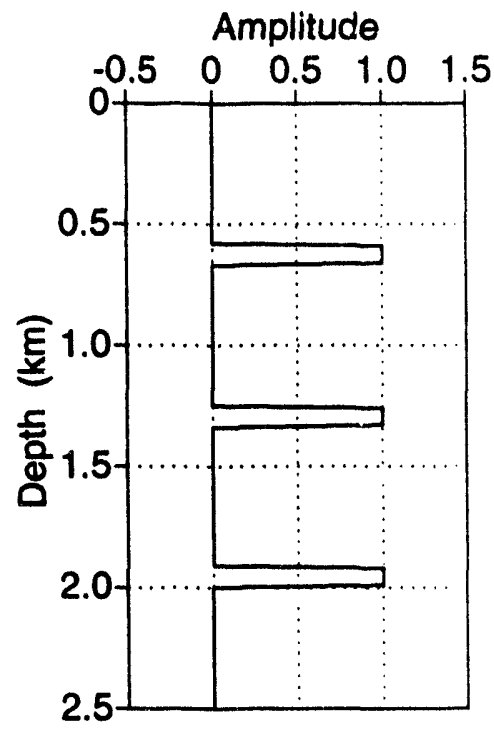

(a)

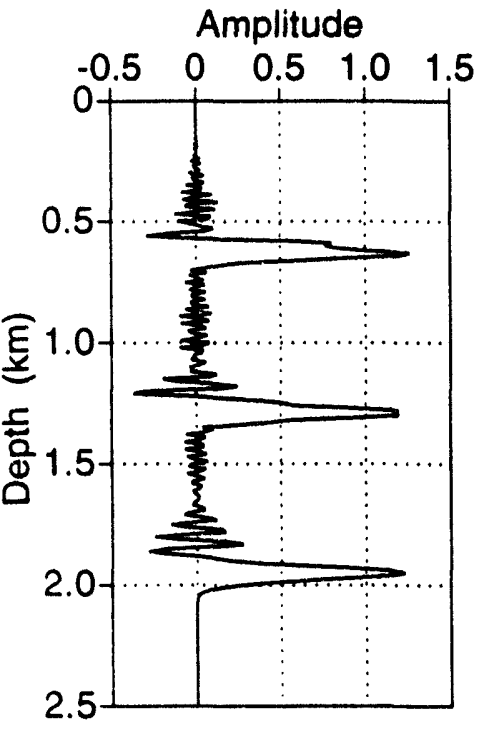

(b)

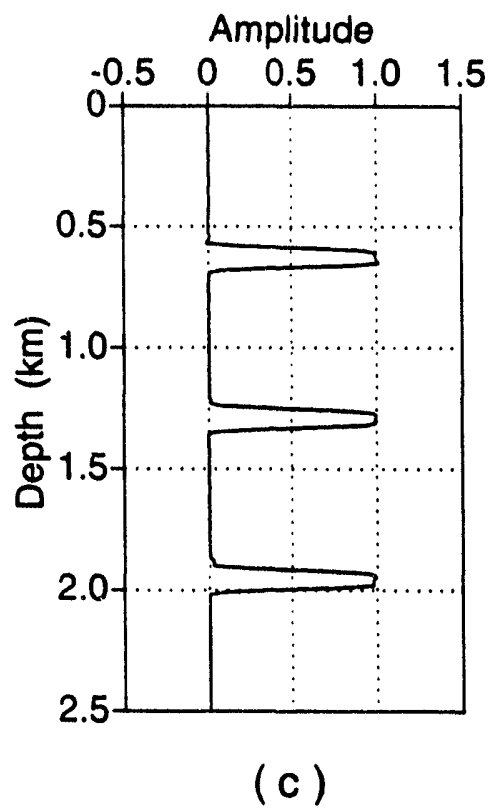

FIG. 4. 


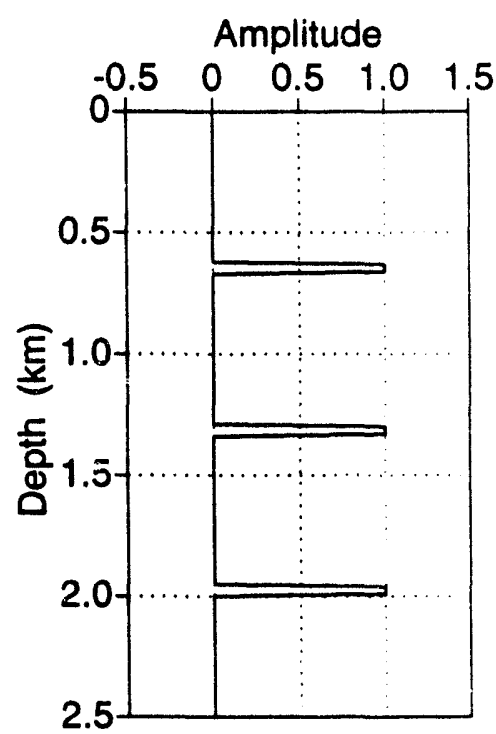

(a)

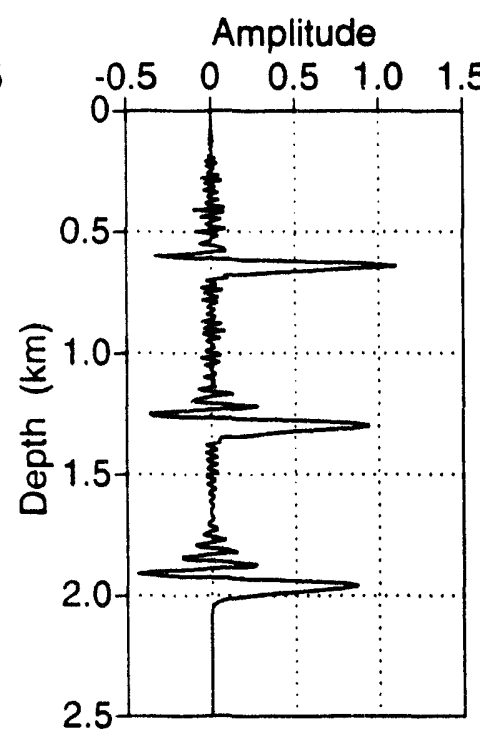

(b)

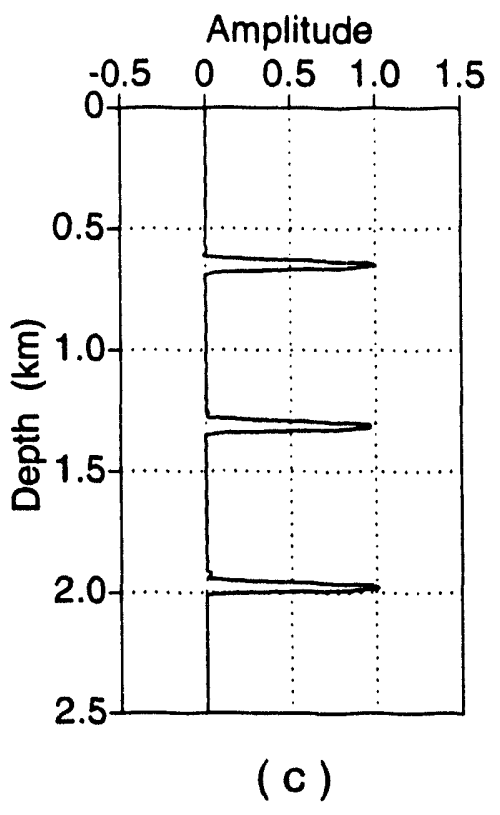

FIG. 5. 


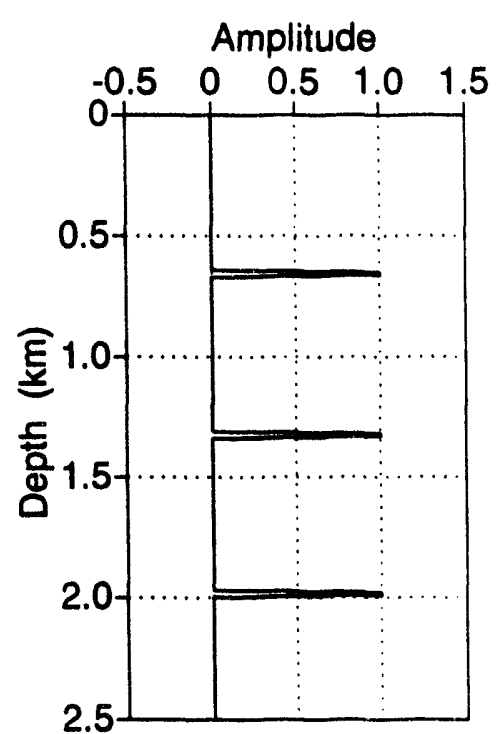

(a)

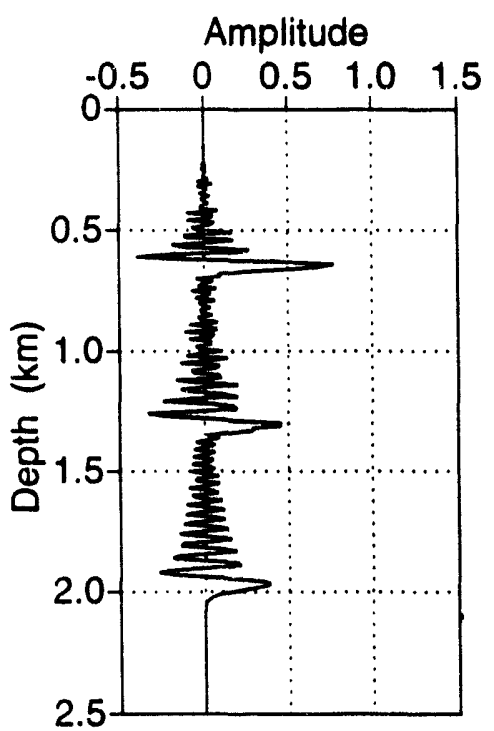

(b)

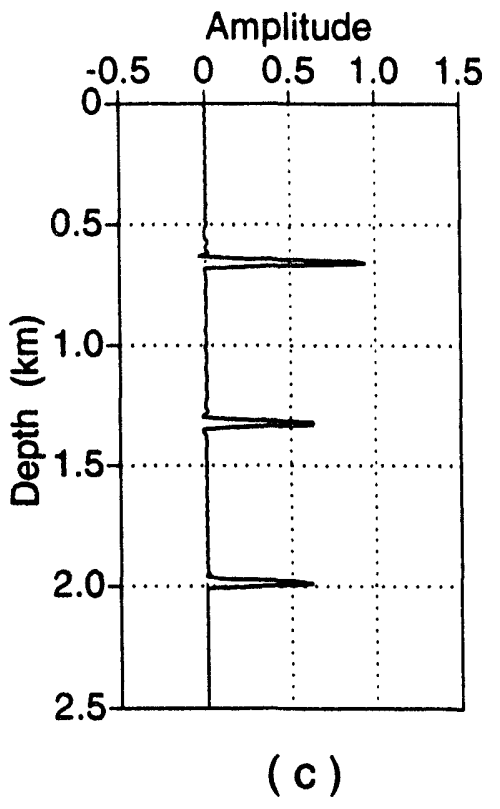

Fig. 6. 
Reflector Model

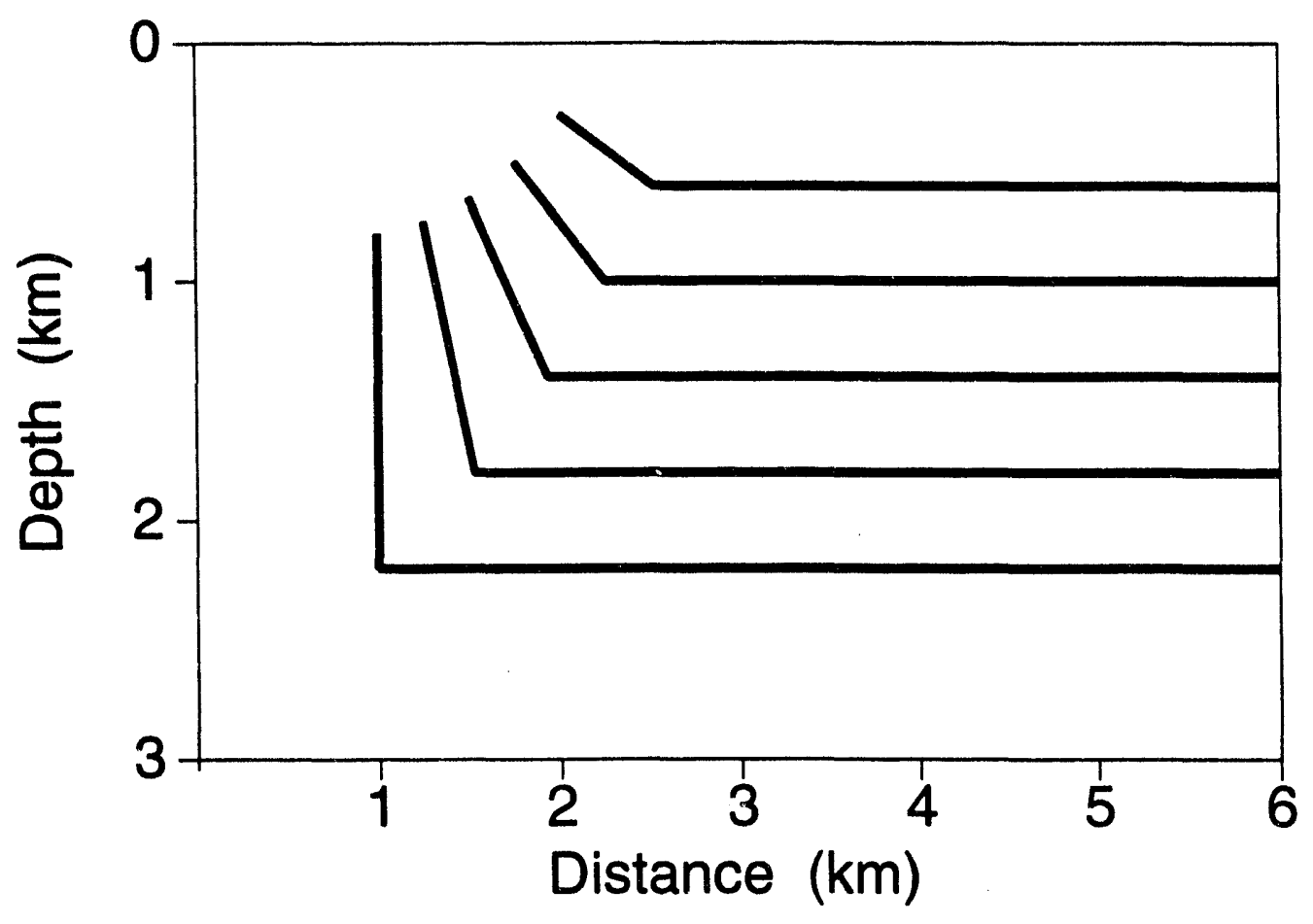

FIG. 7. 


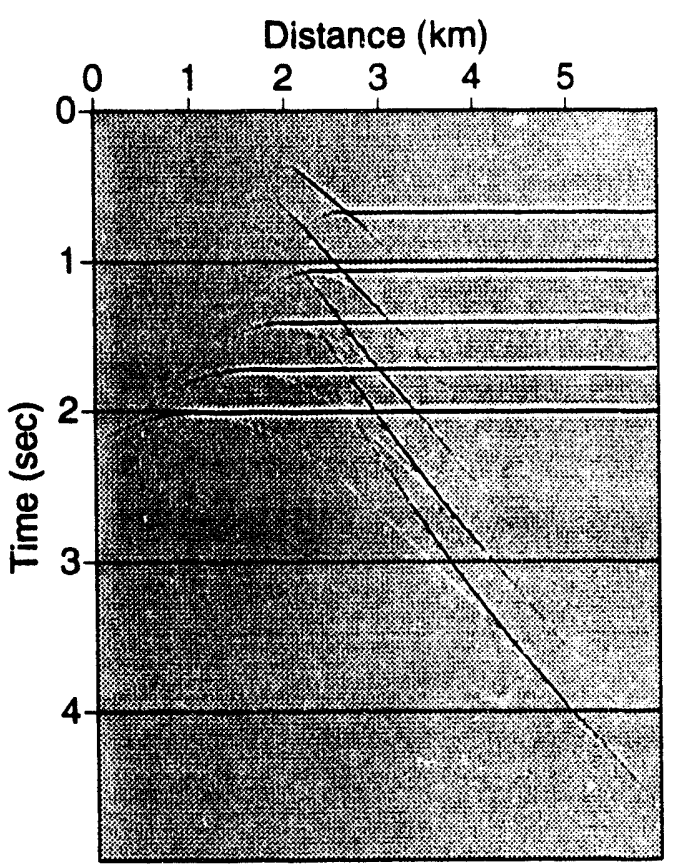

(a) $c(z)=1.6+0.6 z \mathrm{~km} / \mathrm{s}$

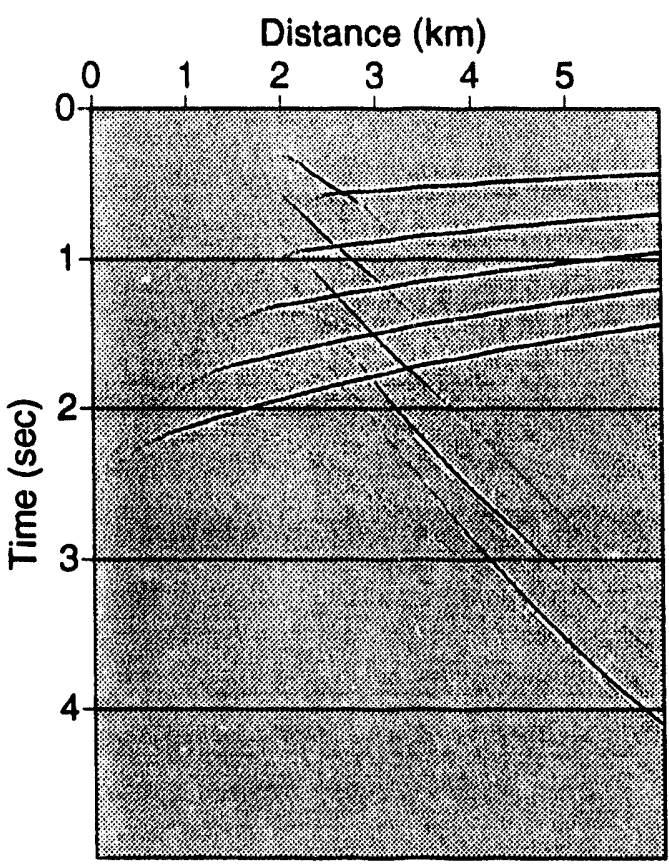

(b) $c(x, z)=1.5+0.2 x+0.35 z \mathrm{~km} / \mathrm{s}$

FIG. 8.

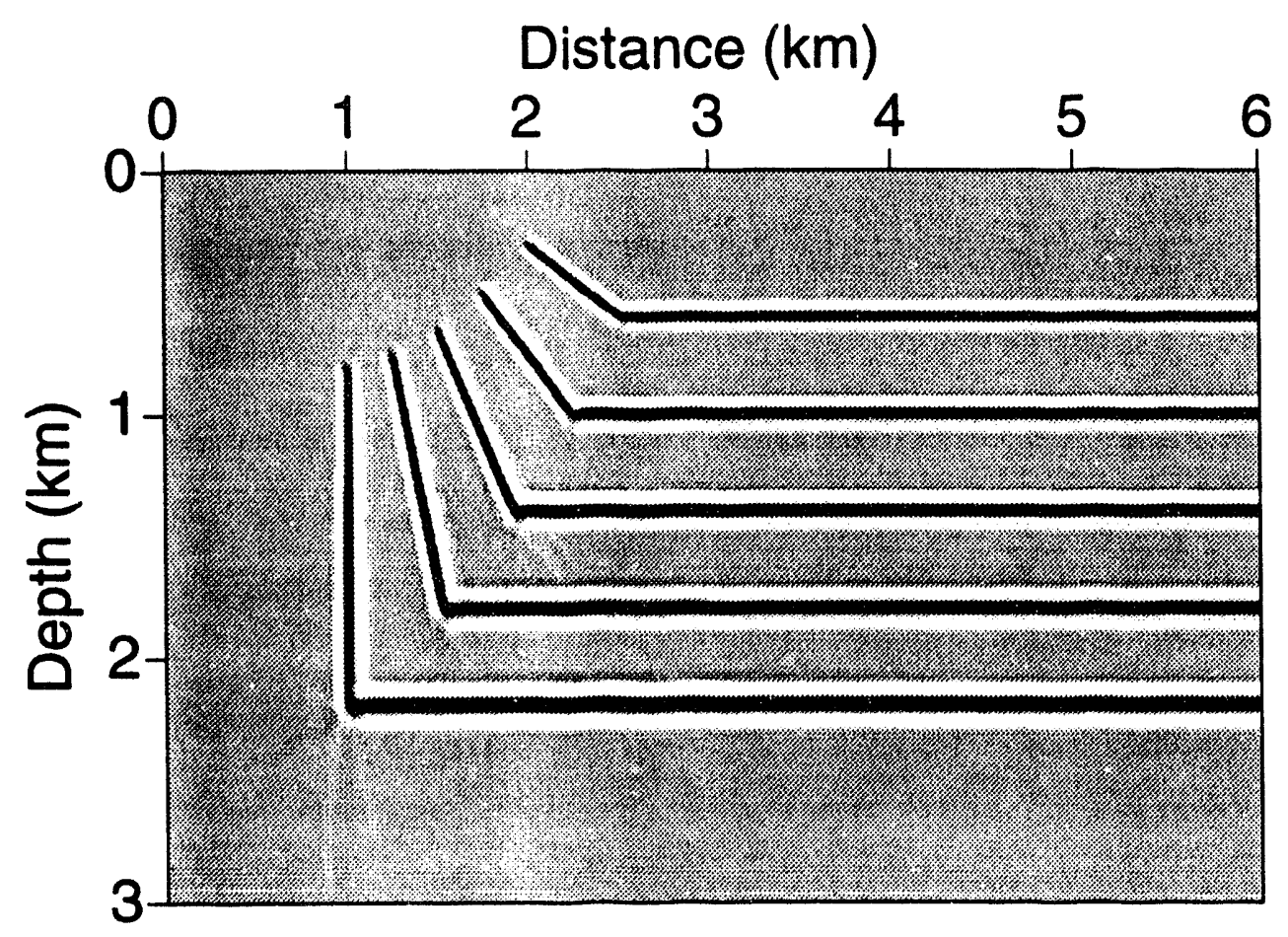

FIG. 9. 


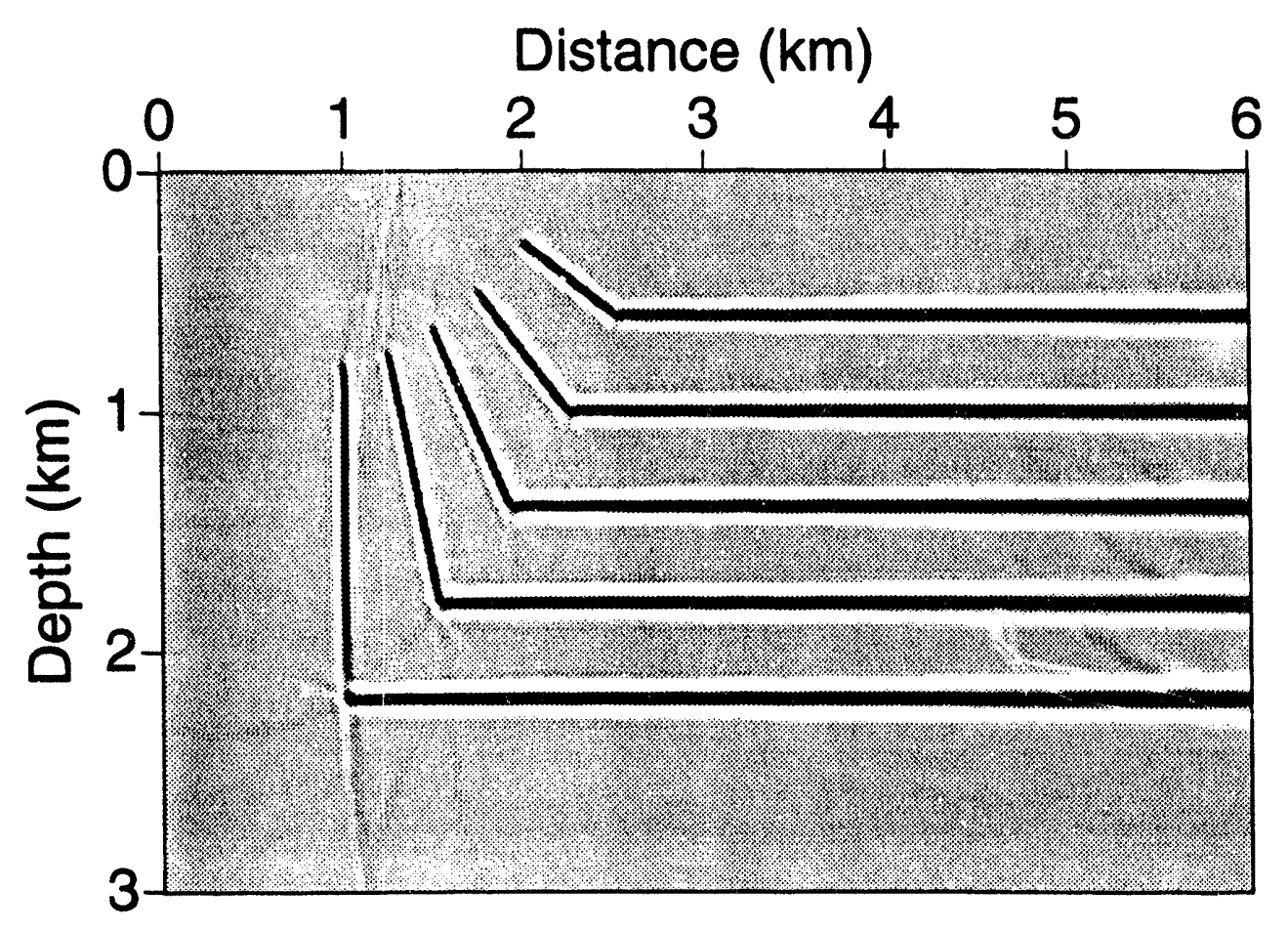

FIG. 10. 


\section{source}

$\mathrm{v}=2.438 \mathrm{~km} / \mathrm{s}$

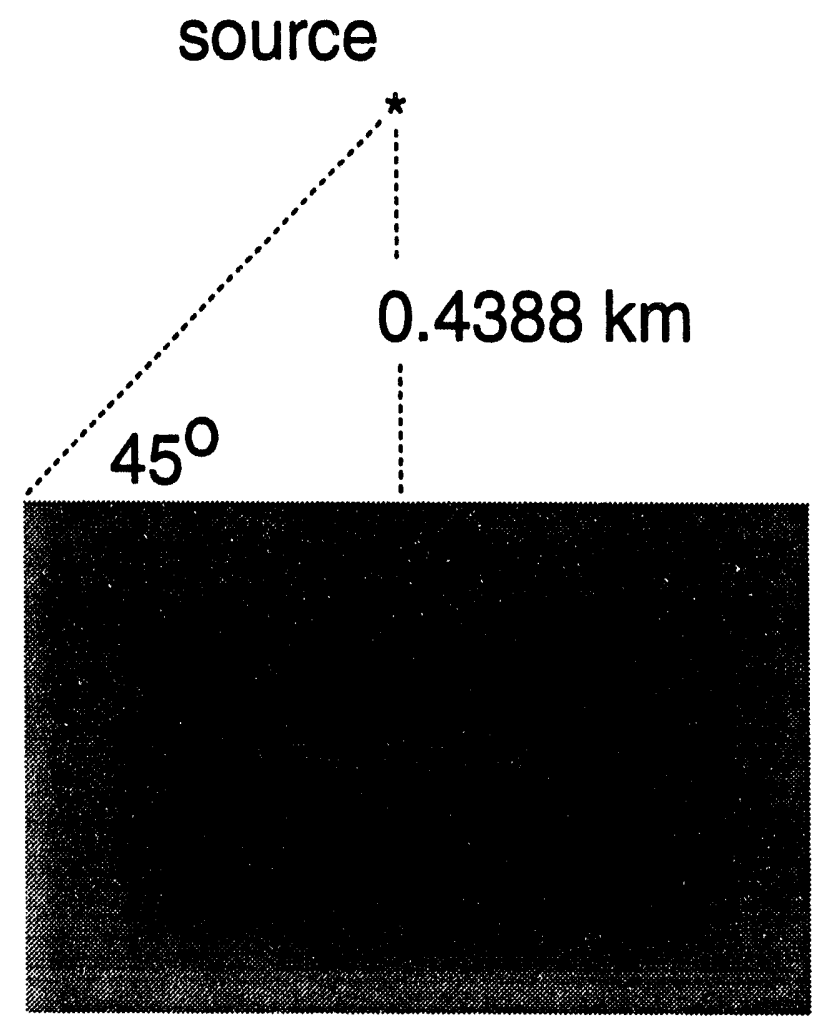

Fig. 11. 


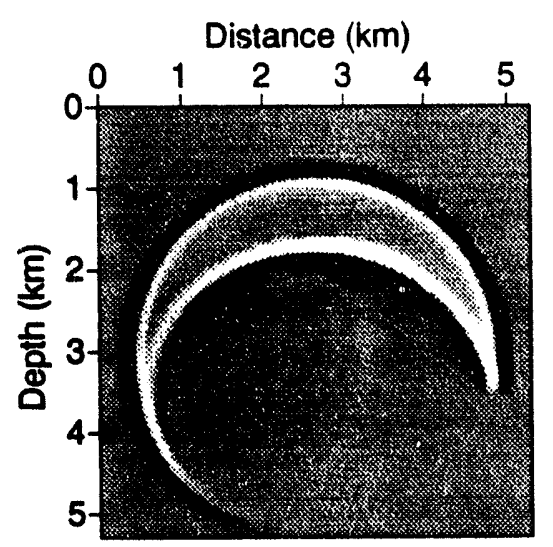

(a)

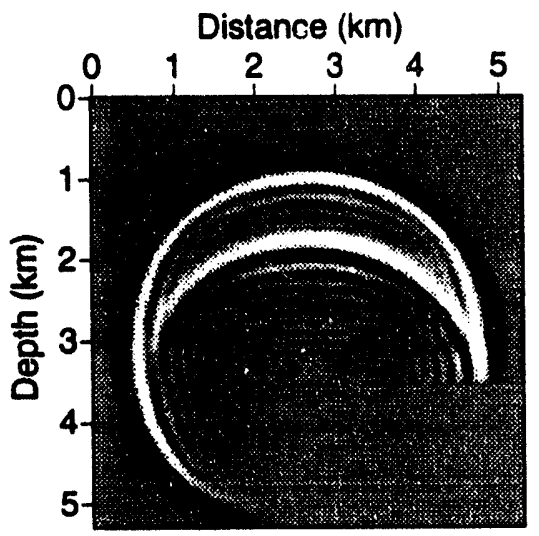

(c)

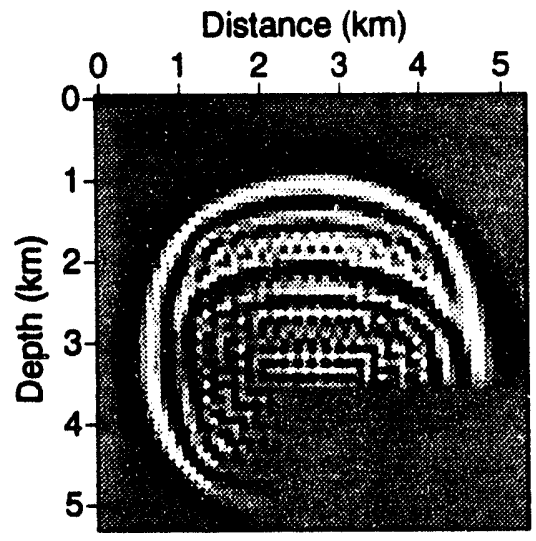

(e)

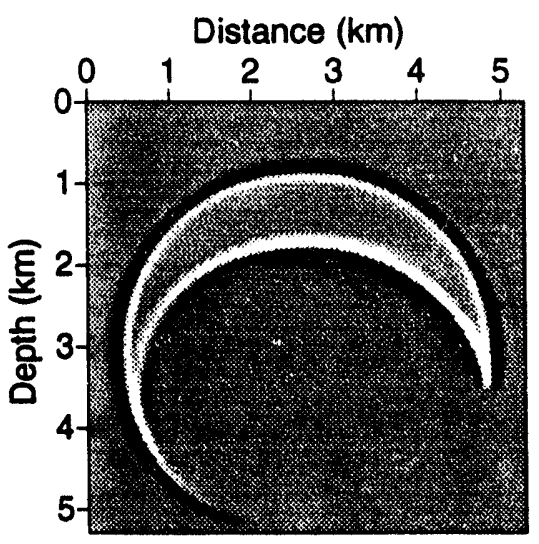

(b)

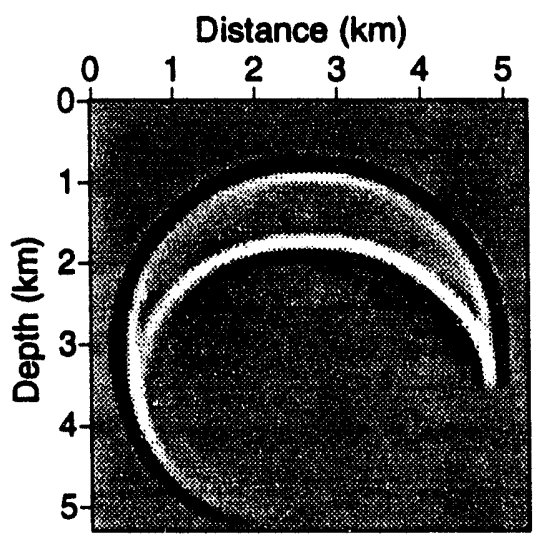

(d)

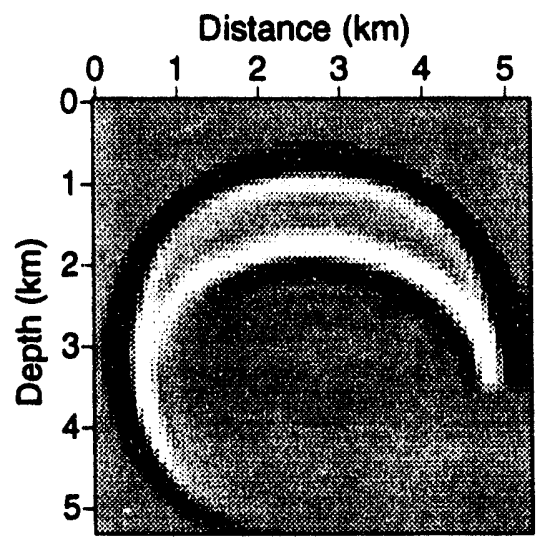

(f)

FIG. 12. 


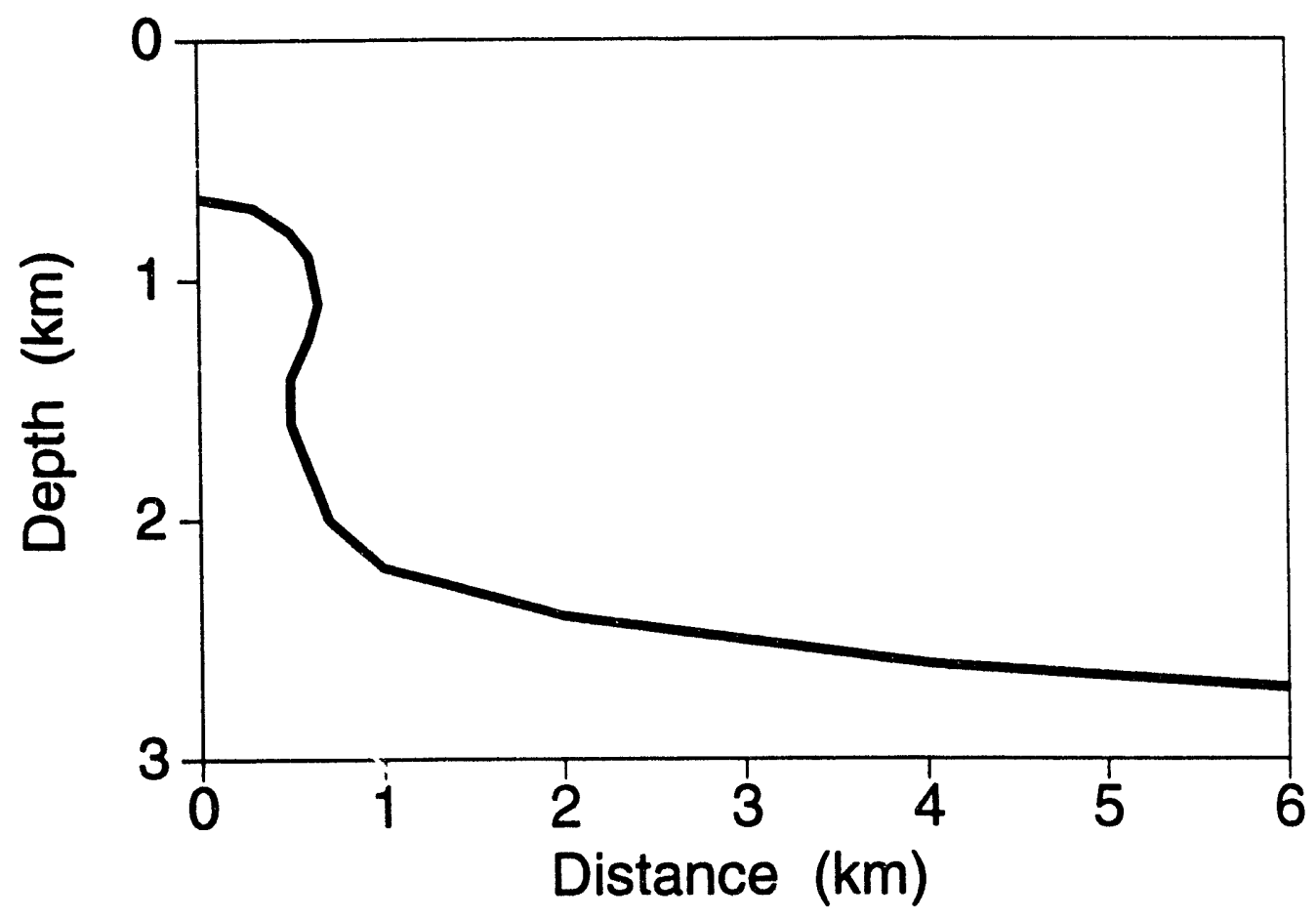

FIG. 13. 


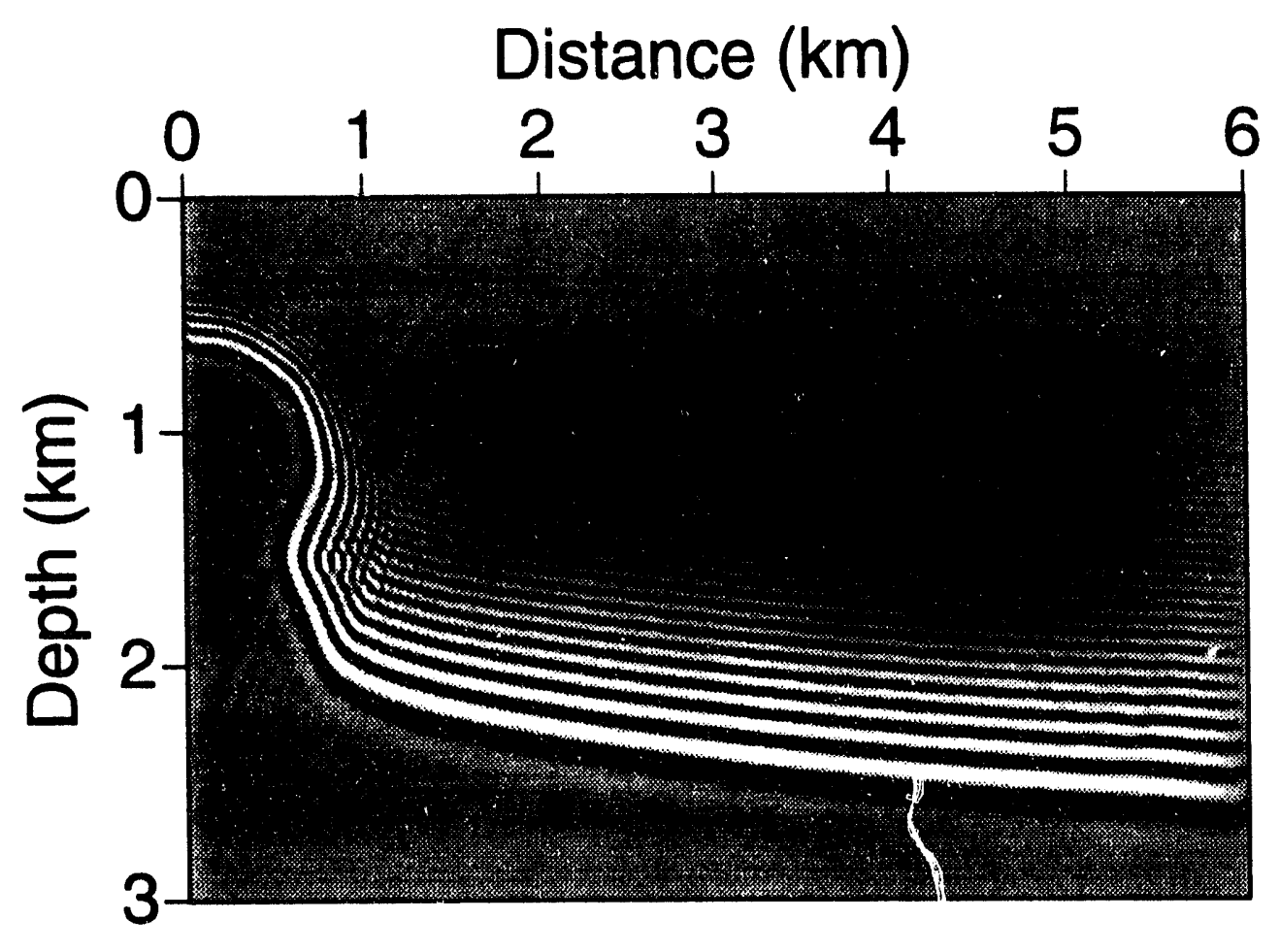

FIG. 14. 


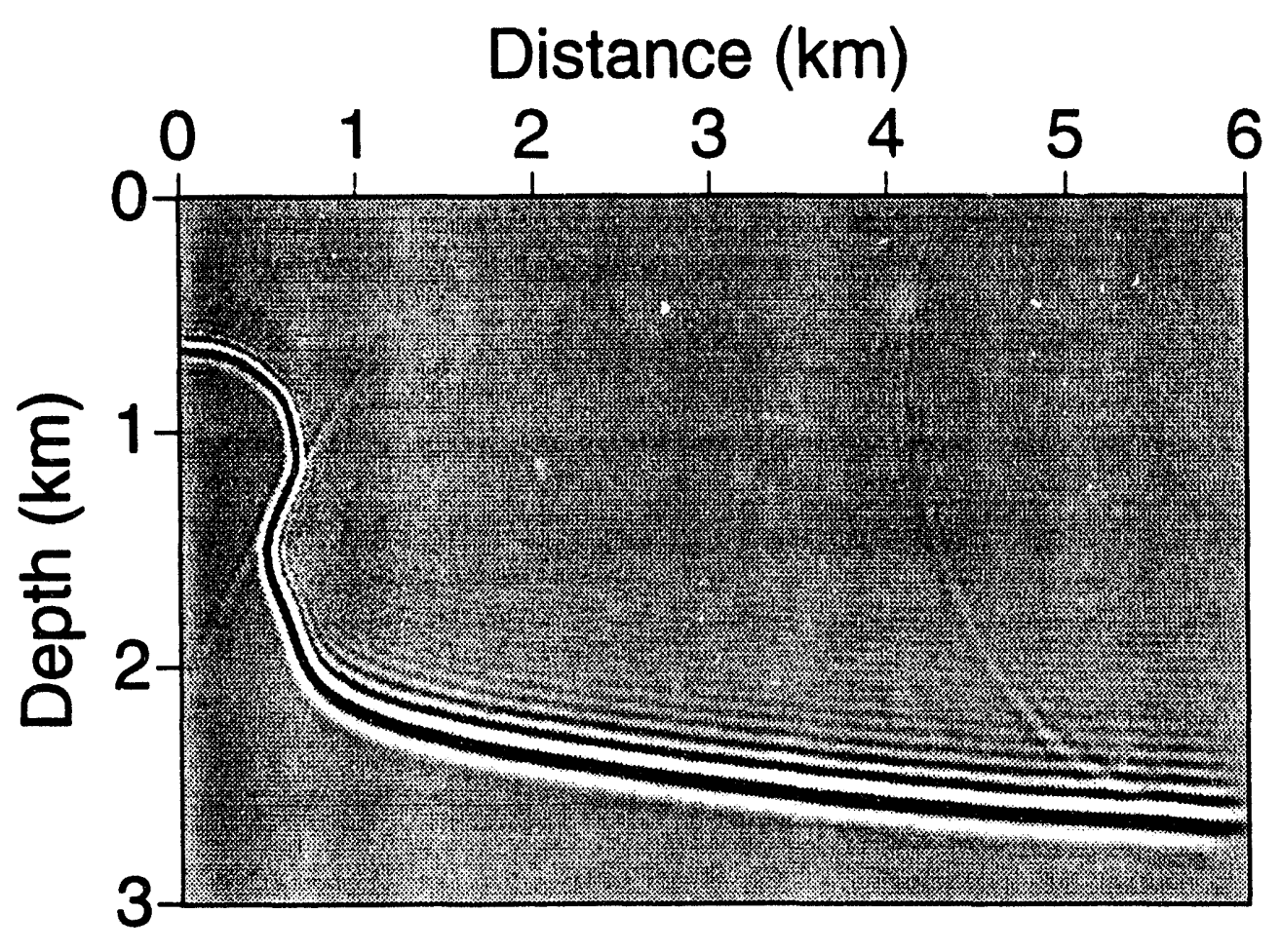

FIG. 15. 


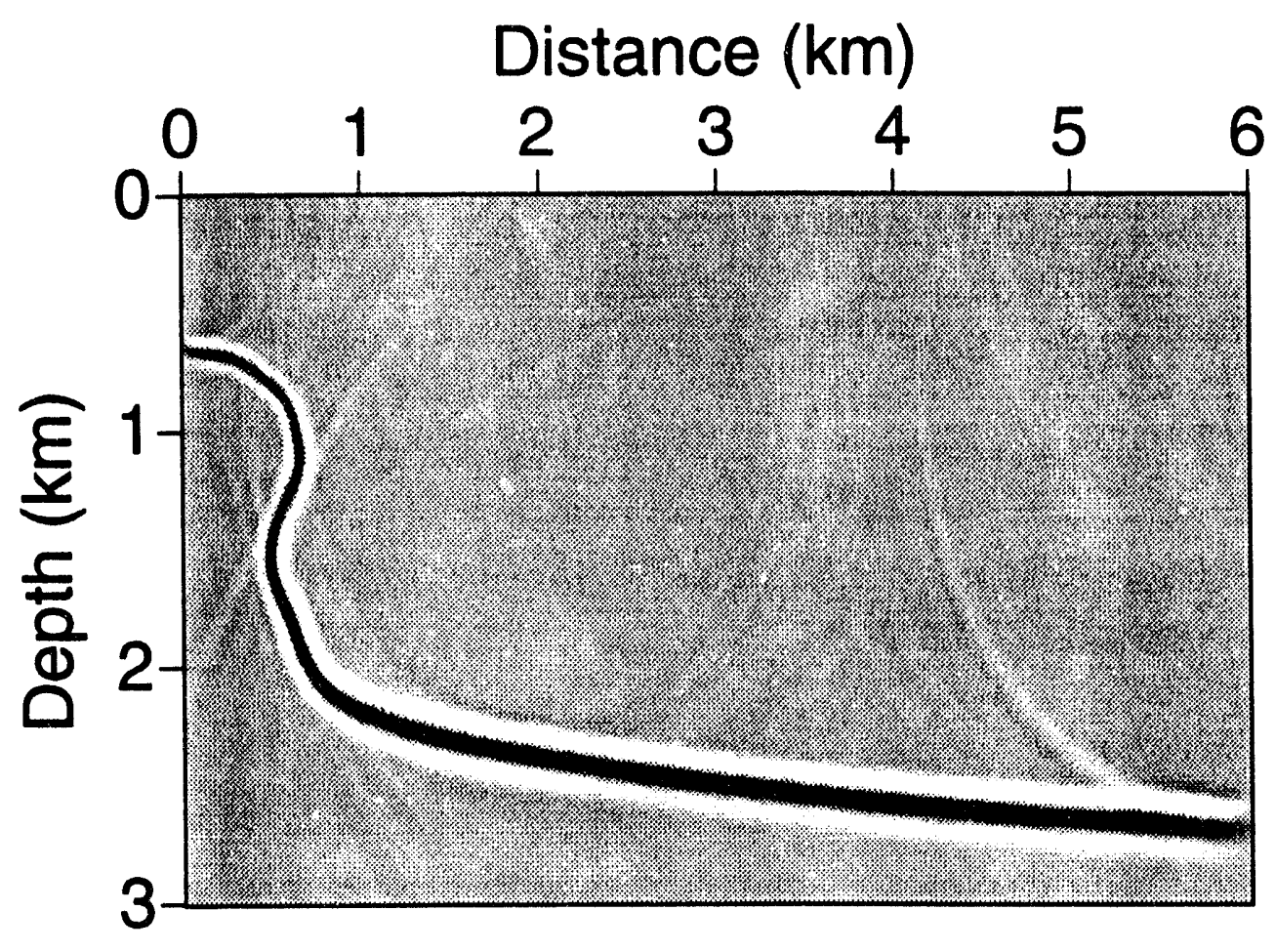

Fig. 16. 


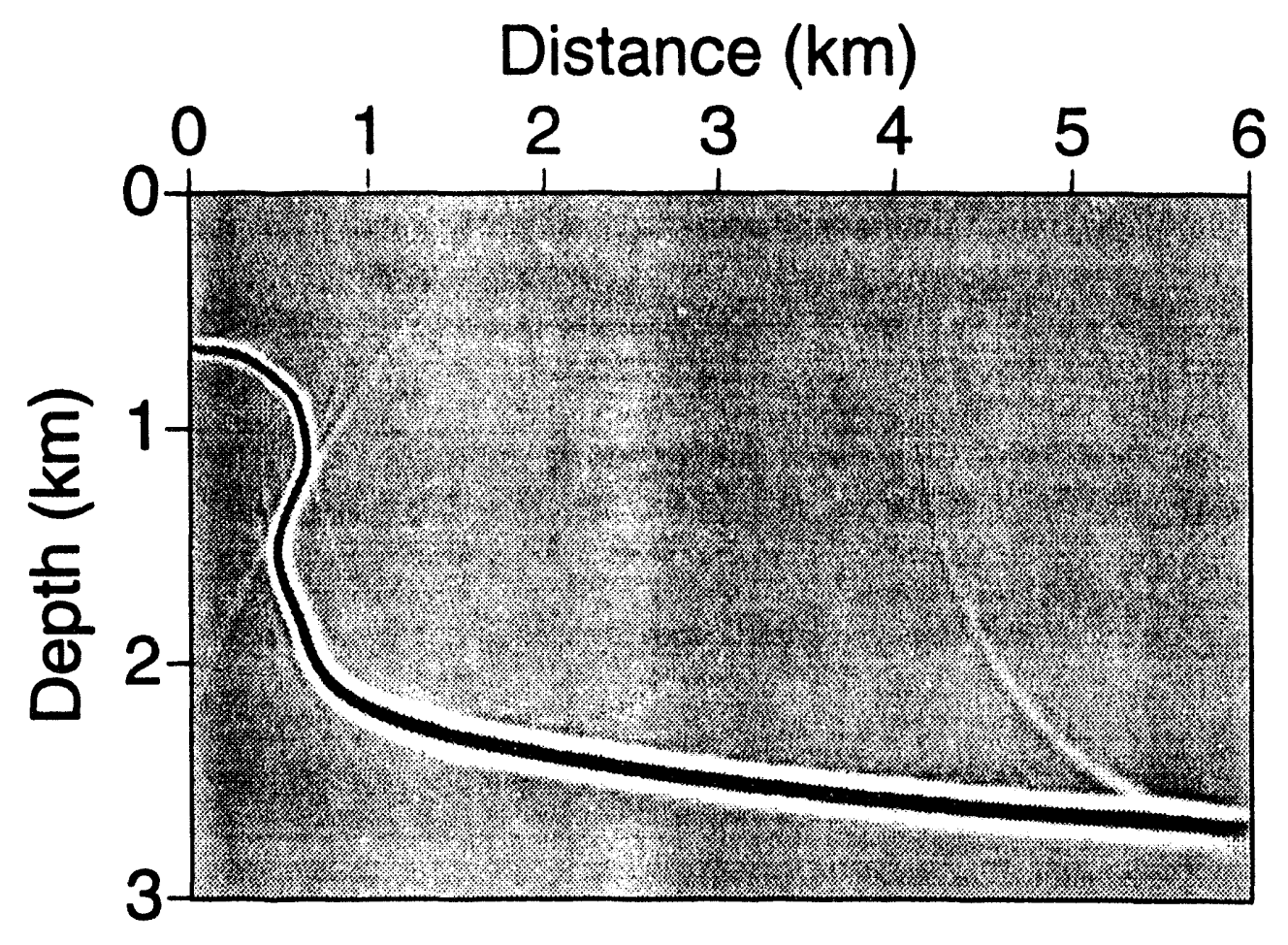

FIG. 17. 


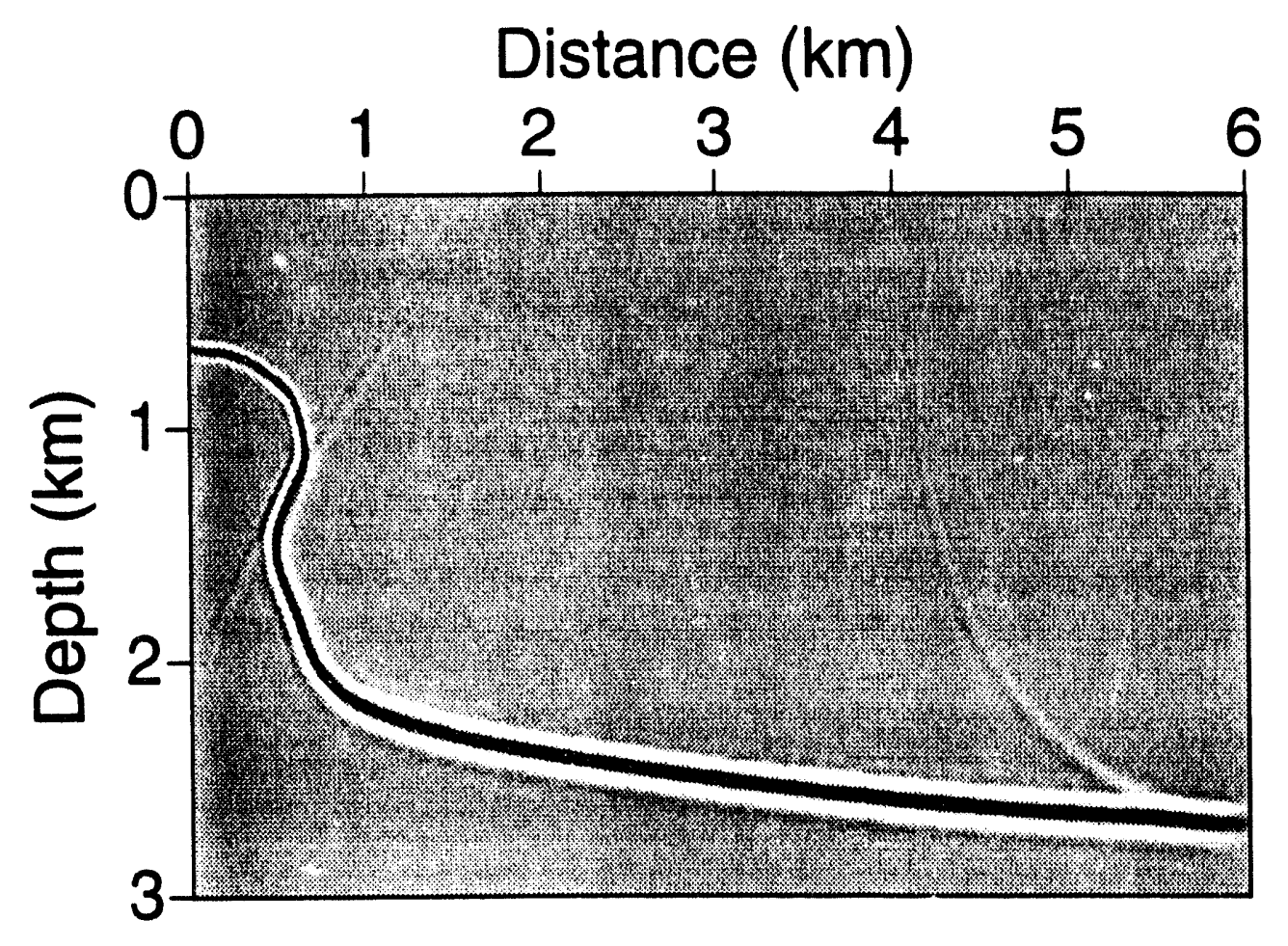

FIG. 18. 


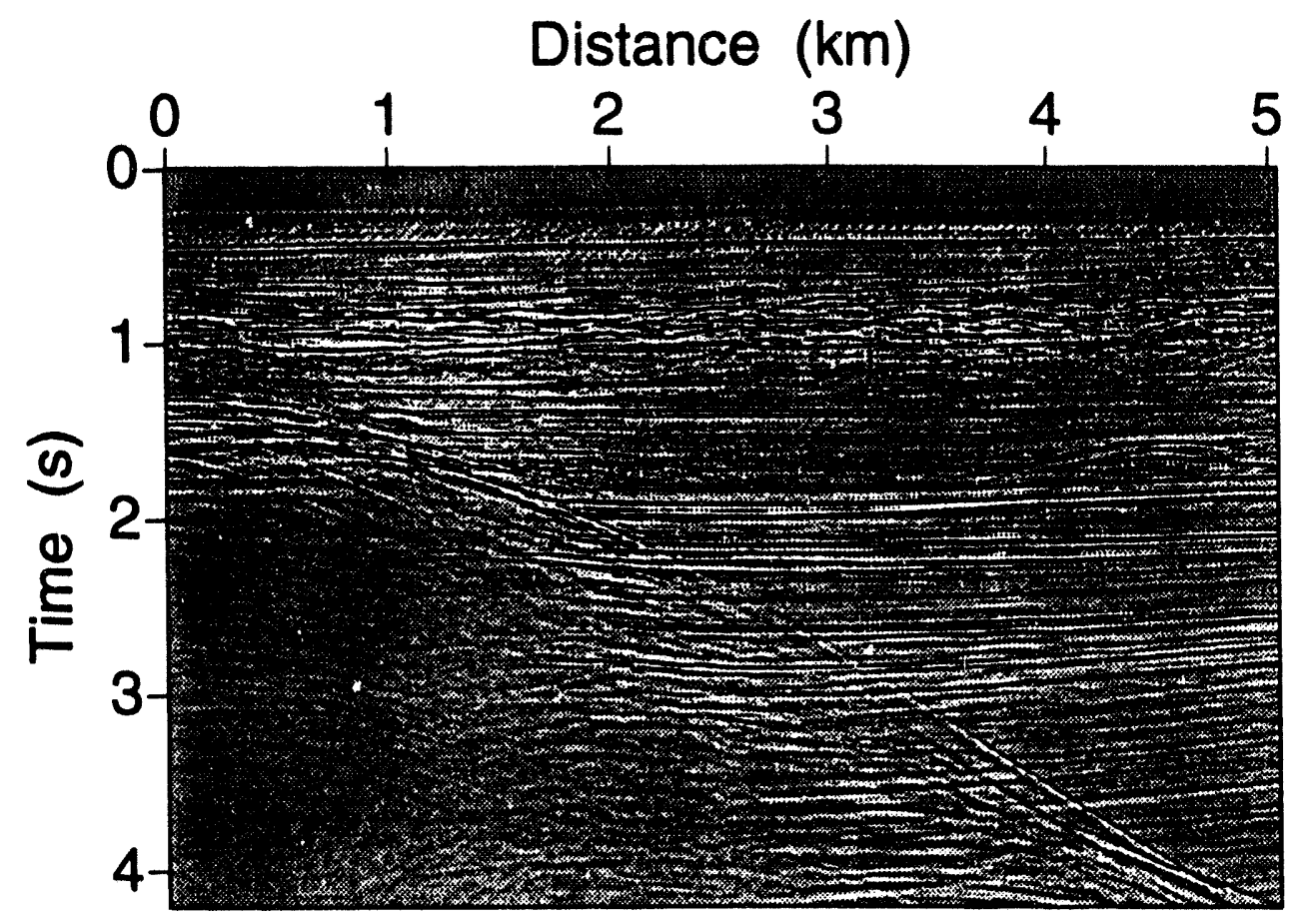

FIG. 19. 


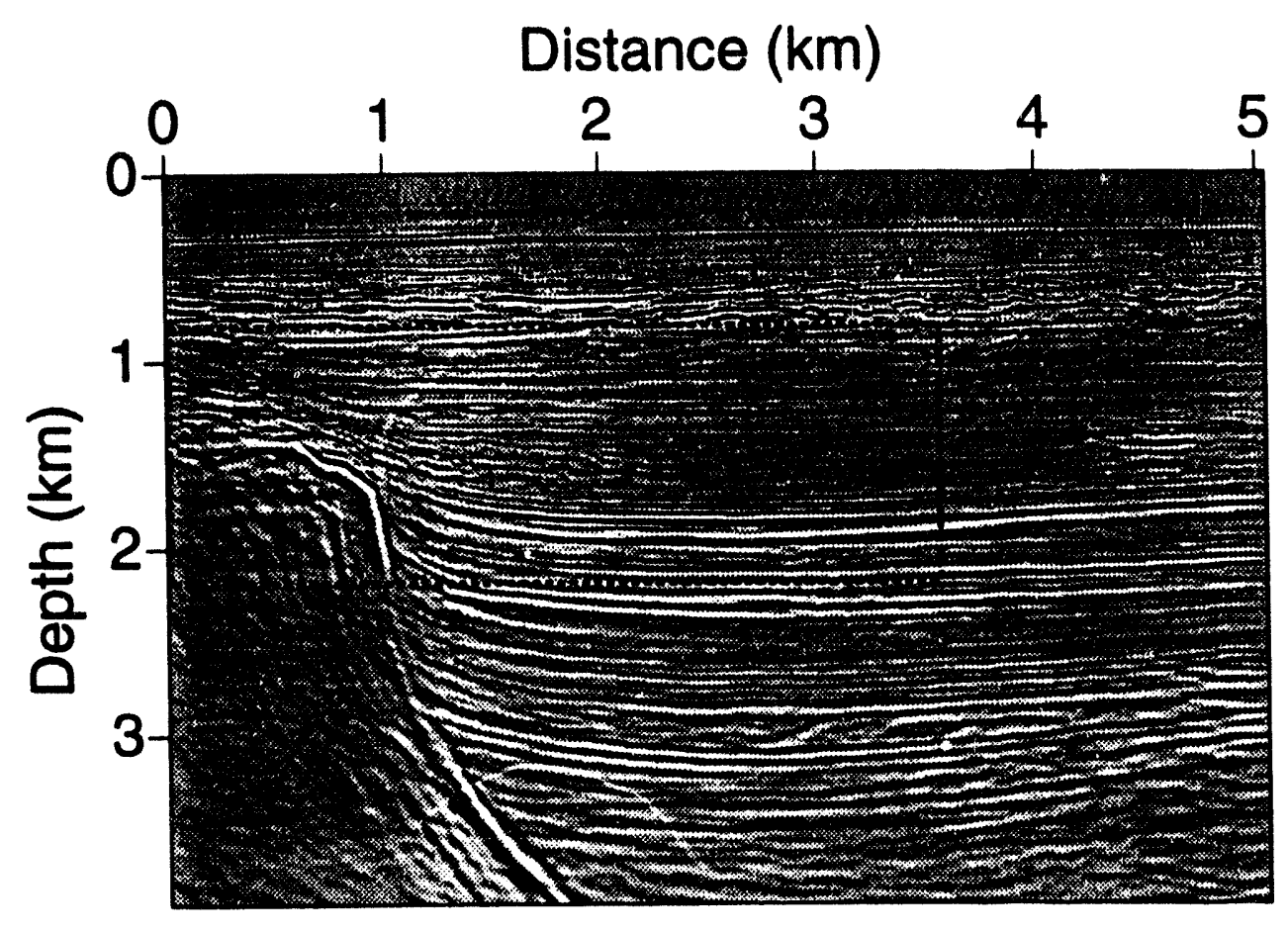

FIG. 20. 


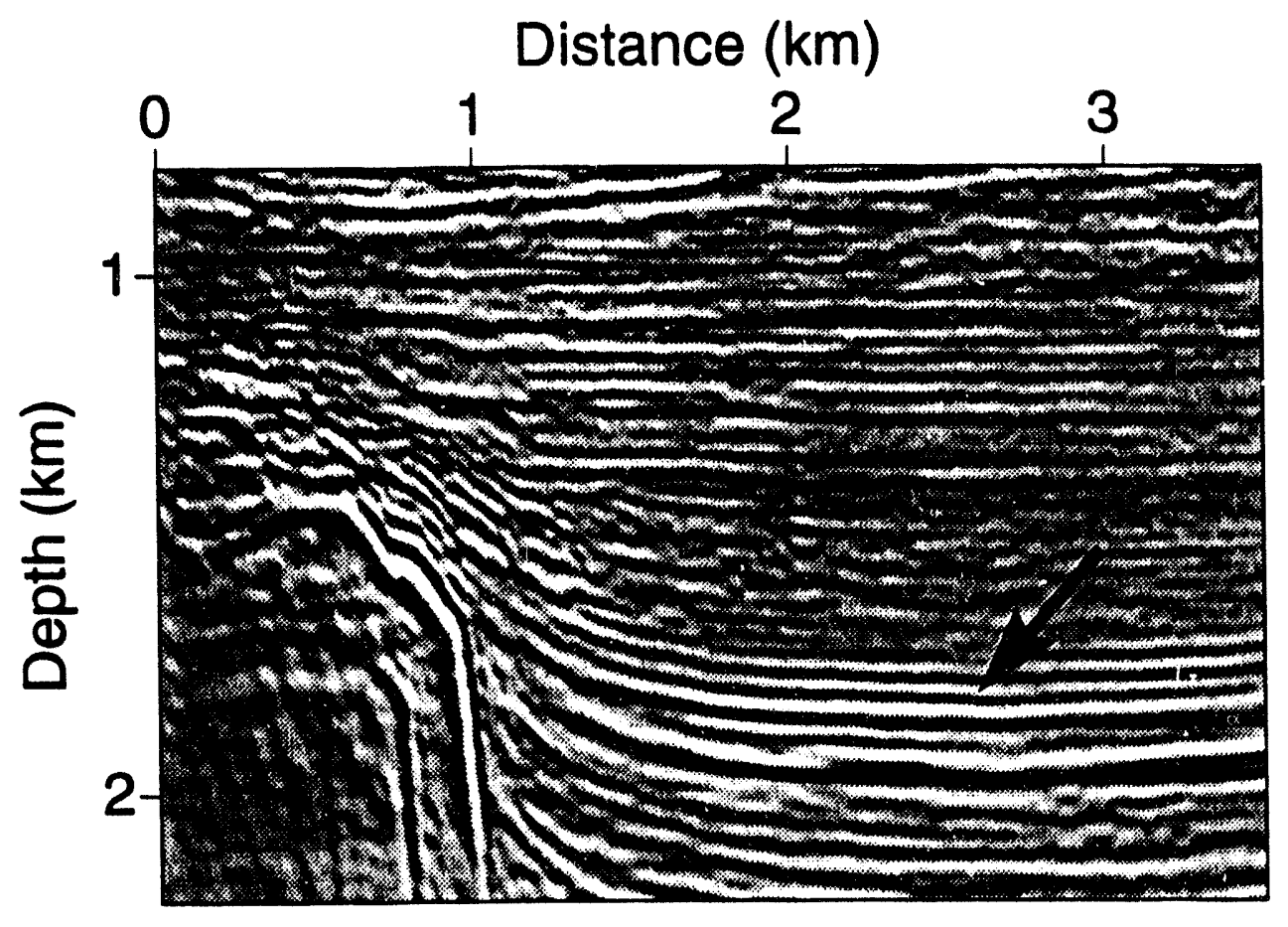

FIG. 21. 


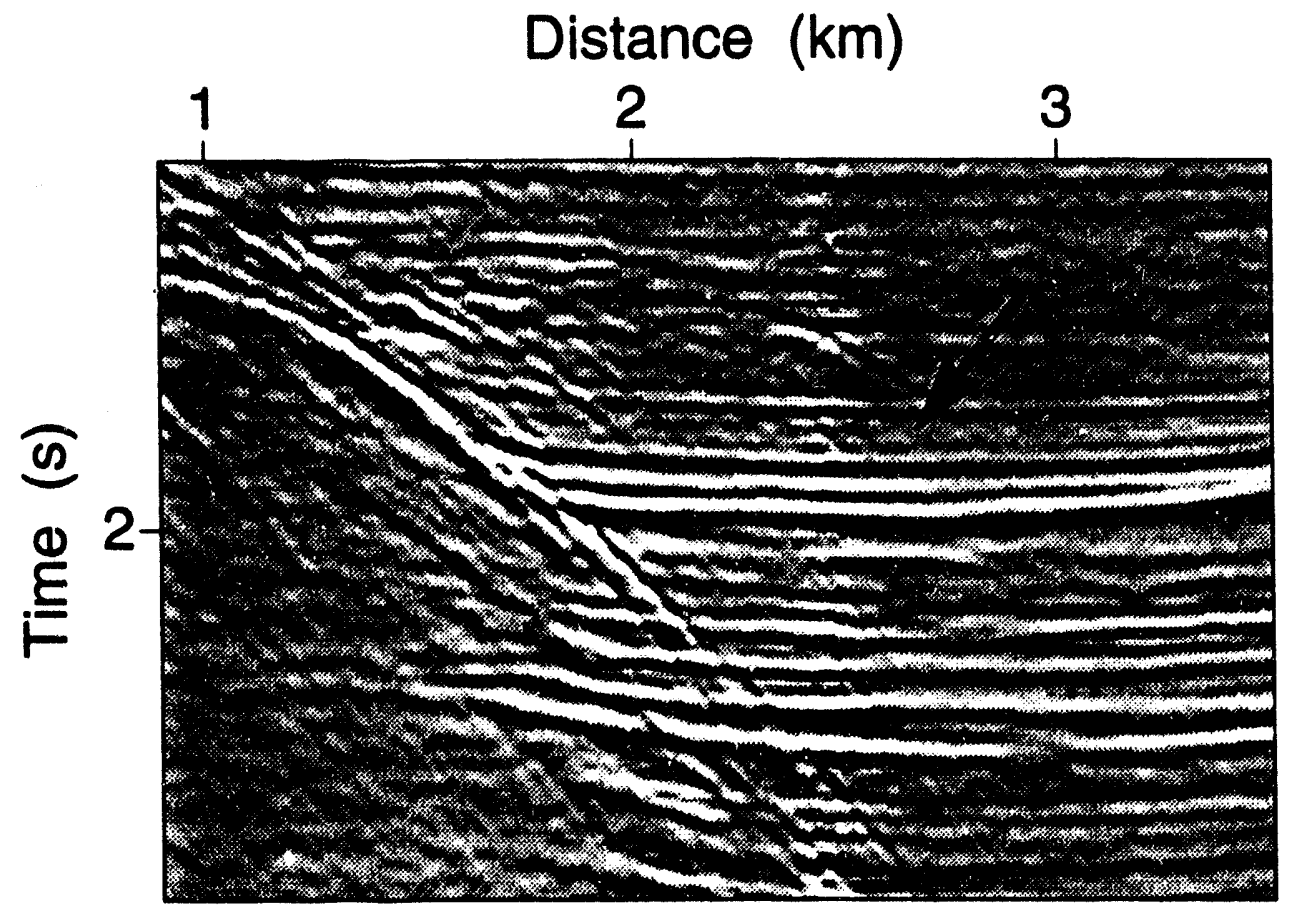

FIG. 22. 


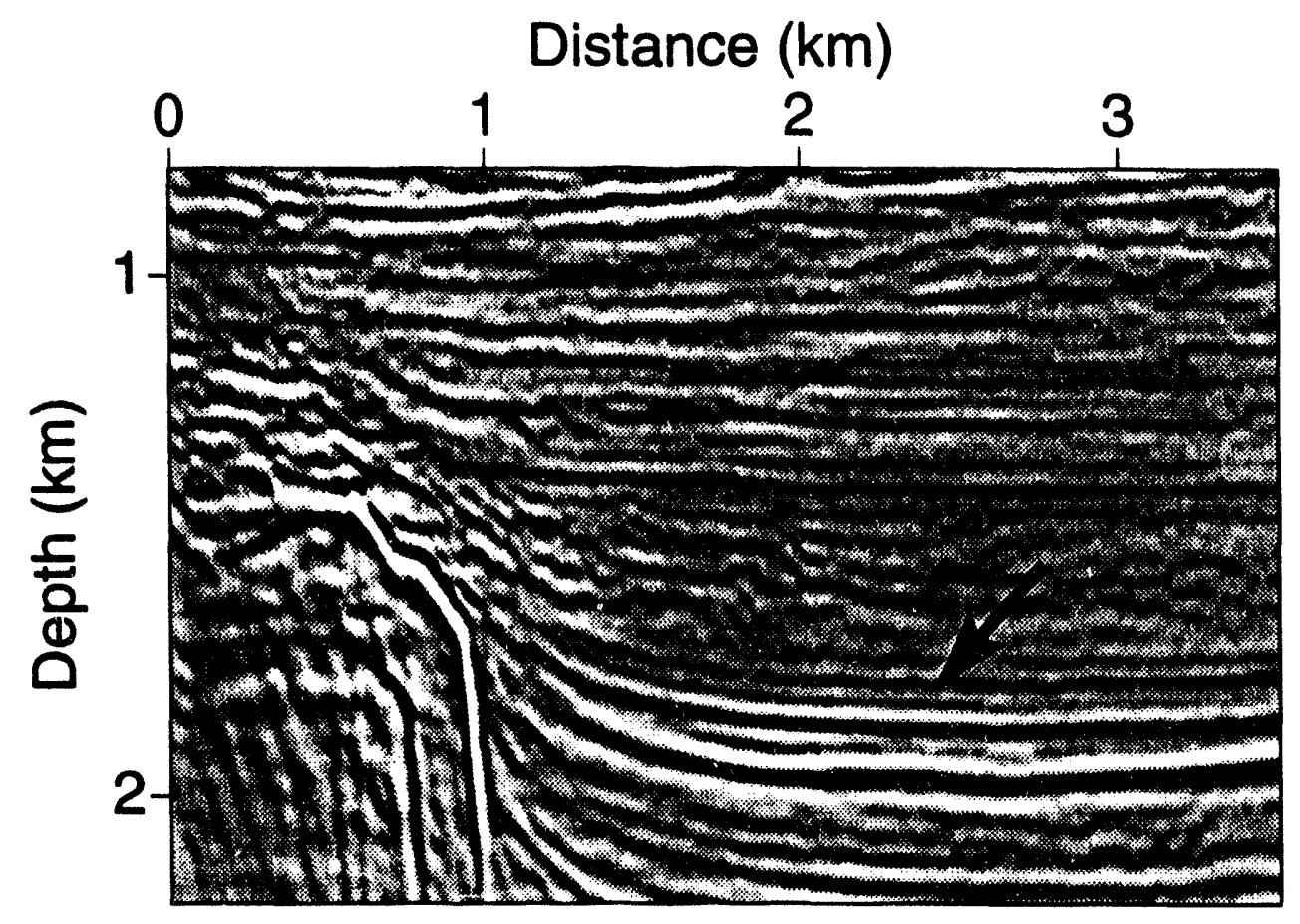

Fig. 23. 

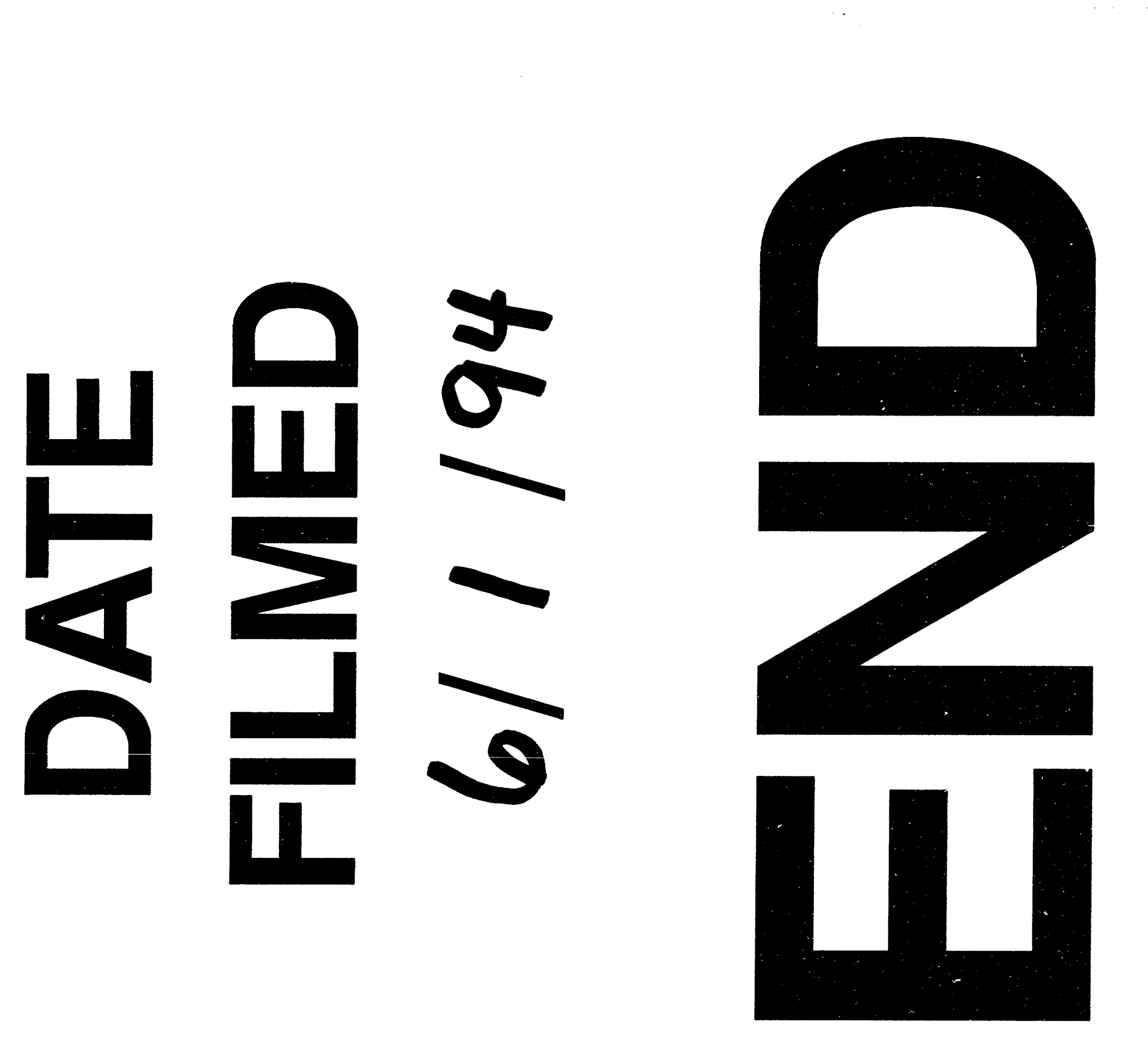